\title{
Triple F-a comet nucleus sample return mission
}

Michael Küppers • H. U. Keller • E. Kührt • M. F. A'Hearn • K. Altwegg •

R. Bertrand - H. Busemann - M. T. Capria - L. Colangeli - B. Davidsson •

P. Ehrenfreund · J. Knollenberg • S. Mottola • A. Rathke · P. Weiss •

M. Zolensky • E. Akim • A. Basilevsky • E. Galimov • M. Gerasimov •

O. Korablev • I. Lomakin • M. Marov • M. Martynov • M. Nazarov •

A. Zakharov - L. Zelenyi - A. Aronica - A. J. Ball - C. Barbieri - A. Bar-Nun •

J. Benkhoff • J. Biele • N. Biver · J. Blum • D. Bockelée-Morvan • O. Botta •

J.-H. Bredehöft • F. Capaccioni • S. Charnley • E. Cloutis · H. Cottin •

G. Cremonese - J. Crovisier • S. A. Crowther • E. M. Epifani • F. Esposito •

A. C. Ferrari - F. Ferri • M. Fulle • J. Gilmour • F. Goesmann • N. Gortsas •

S. F. Green • O. Groussin • E. Grün • P. J. Gutiérrez • P. Hartogh • T. Henkel •

M. Hilchenbach • T.-M. Ho • G. Horneck • S. F. Hviid • W.-H. Ip • A. Jäckel •

E. Jessberger • R. Kallenbach • G. Kargl • N. I. Kömle • A. Korth •

K. Kossacki • C. Krause • H. Krüger • Z.-Y. Li • J. Licandro •

J. J. Lopez-Moreno • S. C. Lowry • I. Lyon • G. Magni • U. Mall • I. Mann •

W. Markiewicz • Z. Martins • M. Maurette - U. Meierhenrich • V. Mennella •

T. C. Ng • L. R. Nittler • P. Palumbo • M. Pätzold • D. Prialnik • M. Rengel •

H. Rickman • J. Rodriguez • R. Roll • D. Rost • A. Rotundi • S. Sandford •

M. Schönbächler • H. Sierks • R. Srama • R. M. Stroud • S. Szutowicz •

C. Tornow - S. Ulamec • M. Wallis · W. Waniak • P. Weissman · R. Wieler •

P. Wurz • K. L. Yung • J. C. Zarnecki

Abstract The Triple F (Fresh From the Fridge) mission, a Comet Nucleus Sample Return, has been proposed to ESA's Cosmic Vision program. A sample return from a comet enables us to reach the ultimate goal of cometary research. Since comets are the least processed bodies in the solar system, the proposal goes far beyond cometary science topics (like the explanation of cometary activity) and delivers invaluable information about the formation of the solar system and the interstellar molecular cloud from which it formed.

M. Küppers $(\varangle) \cdot$ H. U. Keller · F. Goesmann · P. Hartogh · M. Hilchenbach

S. F. Hviid · R. Kallenbach · A. Korth · H. Krüger · U. Mall · W. Markiewicz ·

M. Rengel · R. Roll · H. Sierks

Max-Planck-Institute for Solar System Research,

Max-Planck-Str. 2, 37191 Katlenburg-Lindau, Germany

e-mail: Michael.Kueppers@sciops.esa.int 
The proposed mission would extract three sample cores of the upper $50 \mathrm{~cm}$ from three locations on a cometary nucleus and return them cooled to Earth for analysis in the laboratory. The simple mission concept with a touch-andgo sampling by a single spacecraft was proposed as an M-class mission in collaboration with the Russian space agency ROSCOSMOS.

Keywords Comets $\cdot$ Cosmogony $\cdot$ Sample return $\cdot$ Space mission

\section{Introduction}

At the first stage of the formation of the solar system there was the solar/protoplanetary nebula collapsing from a molecular cloud. The central star-our Sun-formed and started to heat the dust/gas mixture. Dust particles sank to the mid-plane, accreted, and agglomerated to planetesimals and cometesimals, the building blocks of the planets. At the outer fringes of the nebula the temperatures were cold enough that ices persisted and volatiles condensed before the comets were formed. Investigating the chemical and physical properties of this primordial mixture is a key to understanding how our solar system formed-and ultimately how life has started.

The primordial mixture has been preserved-almost unaltered from further processing due to high speed impacts, gravitational compression and

\section{Küppers}

European Space Astronomy Centre, P.O. Box 78,

28691 Villanueva de la Cañada, Madrid, Spain

E. Kührt · J. Knollenberg · S. Mottola · J. Benkhoff · J. Biele · N. Gortsas ·

G. Horneck · C. Krause · C. Tornow

German Aerospace Center (DLR), Berlin, Germany

M. F. A'Hearn

University of Maryland, College Park, MD, USA

K. Altwegg · A. Jäckel · P. Wurz

University of Berne, Berne, Switzerland

R. Bertrand

CNES, Toulouse, France

H. Busemann · A. J. Ball · S. F. Green · J. C. Zarnecki

Open University, Milton Keynes, UK

M. T. Capria · F. Capaccioni · G. Magni

IASF/INAF, Rome, Italy

L. Colangeli · E. M. Epifani · F. Esposito · V. Mennella

INAF-Osservatorio Astronomico di Capodimonte, Naples, Italy

B. Davidsson · H. Rickman

University of Uppsala, Uppsala, Sweden 
heating - in low density cometary nuclei whose temperature did not exceed $50 \mathrm{~K}$. Sophisticated analyses of this material in our laboratories will allow us to determine the ratio of processed to original interstellar material, and to determine the time scales of grain formation. Key questions like 'How important was ${ }^{26} \mathrm{Al}$ for heating even small bodies in the first millions of years?' can be assessed by determining the time scale for accretion of cometesimals as well as the structure of the cometesimals. Investigation of cometary material provides information about the original (primordial) mixture out of which the planetesimals and hence planets formed before they were altered in this formation process. The proposed Triple F (Fresh From the Fridge), a Comet Nucleus Sample Return (CNSR) mission, concentrates on retrieving samples of this original mixture to bring them back for analyses that can only be undertaken in terrestrial laboratories. There the detailed chemical and isotopic composition and the internal structure of ice-mineral cometary grains will be measured as well as the granulation of the volatile material.

The relevance of cometary research goes far beyond the investigation of minor bodies, of their physical and chemical properties or even of how they came about. The driving quest has always been to learn about the composition of the primordial nebula mixture and the formation of our solar system. Now is the time to achieve this ultimate goal of the European space programme that started more than 20 years ago with ESA's first planetary mission to fly-by comet Halley. The stepwise preparation by cometary fly-bys (VEGAs, Giotto, Sakigake, and Suisei fly-bys of 1P/Halley [53], Deep Space

P. Ehrenfreund

University of Leiden, Leiden, Netherlands

A. Rathke

EADS Astrium, Friedrichshafen, Germany

P. Weiss · T. C. Ng · K. L. Yung

The Hongkong Polytechnic University, Hong Kong, China

M. Zolensky

NASA Johnson Space Center, Houston, USA

E. Akim · M. Marov

Keldysh Institute, Moscow, Russia

A. Basilevsky · E. Galimov · M. Nazarov

Vernadskij Institute, Moscow, Russia

M. Gerasimov · O. Korablev · A. Zakharov · L. Zelenyi

Space Research Institute, Moscow, Russia

I. Lomakin · M. Martynov

Lavochkin Association, Moscow, Russia

A. Aronica $\cdot$ P. Palumbo $\cdot$ A. Rotundi

University of Naples, Naples, Italy 
1 fly-by of 19P/Borrelly [46]), impacts (Deep Impact on 9P/Tempel 1, [2]), collection of dust (Stardust at 81P/Wild 2, [10]), and a rendezvous (Rosetta with 67P/Churyumov-Gerasimenko, [27]) leads to an understanding of comets and their spectacular activity. This knowledge now provides a firm basis for the design and successful execution of a sample return mission.

The Rosetta mission will investigate the cometary nucleus from orbit and also by instruments placed onto the surface of comet 67P/ChuryumovGerasimenko (CG) by a lander. These investigations will take place in the year 2014. The physical characteristics of the nucleus of CG will be investigated in detail, its coarse chemical composition will be analysed, and the physics of its activity examined-a very major step forward in our understanding of the chemistry and physics of cometary nuclei. However, many questions about the formation of the planetary system will not be answered. How was the interstellar (molecular cloud) material metamorphosed into solar system compounds, organics and minerals? Did comets contribute to the development of life on Earth? Investigations to reveal the physical and chemical processes and their time scales during the early stages of planetary formation need analyses on ppb levels that cannot be realized by Rosetta. Addressing such questions has successfully been demonstrated on meteoritic samples from various types of asteroids, the Moon, and Mars. The recent analysis of nonvolatile material collected during the Stardust fly-by of comet 81P/Wild 2 shows that microscopic high temperature material formed near the early Sun can be found in cometary nuclei that formed at low temperatures, possibly

C. Barbieri · G. Cremonese · F. Ferri

University of Padova, Padova, Italy

A. Bar-Nun · D. Prialnik

University of Tel-Aviv, Tel Aviv, Israel

N. Biver · D. Bockelée-Morvan · J. Crovisier

Paris Observatory, Meudon, France

J. Blum

Technical University Braunschweig, Braunschweig, Germany

O. Botta

International Space Science Institute, Berne, Switzerland

J.-H. Bredehöft

University of Bremen, Bremen, Germany

S. Charnley

NASA Ames Research Center, Mountain View, USA

E. Cloutis

University of Winnipeg, Winnipeg, Canada

H. Cottin

University of Paris, Paris, France 
as low as $30 \mathrm{~K}$, in the outer reaches of the solar system [42]. Although an evolutionary explanation has not yet been ruled out, the structure and the chemical heterogeneity of 9P/Tempel 1 as observed by Deep Impact suggest that large cometesimals may contain materials from different parts of the protoplanetary disk [6]. How well, on what time scales, and how far out was the solar nebula mixed before the building blocks of the planets have accreted and agglomerated? Many of these physical and chemical processes have been revealed by the interpretation of the extremely sophisticated analyses of the diverse meteoritic materials. However, a global and conclusive picture and time line for the formation process of our solar system has not yet been developed. Bringing back a sample from a body that formed on the fringes of the planetary system, the temperature of which was always low enough to trap compounds as volatile as $\mathrm{CO}$ or $\mathrm{CH}_{4}$ will allow us to investigate an end member of the minor bodies of the planetary system. Here we have the best chance to understand the relationship between the original interstellar material of the collapsing molecular cloud and the processed end-products found in the meteoritic samples. The high content of volatiles in cometary nuclei shows that little processing occurred during the formation of the comet or of the parent body it broke up from.

The scientific rationale for the Triple F mission is outlined in Section 2 on the basis of recent results from the cometary missions Deep Impact and Stardust. Cometary nucleus properties will be very well understood from investigations by Rosetta's Lander. The months spent near the nucleus from the onset of its

S. A. Crowther · J. Gilmour · T. Henkel · I. Lyon · D. Rost
University of Manchester, Manchester, UK

A. C. Ferrari

University of Cambridge, Cambridge, UK

M. Fulle

Trieste Observatory, Trieste, Italy

O. Groussin

LAM, Marseille, France

E. Grün · R. Srama

MPIfK, Heidelberg, Germany

P. J. Gutiérrez · J. J. Lopez-Moreno · J. Rodriguez

IAA-CSIC, Granada, Spain

T.-M. Ho

ESA-ESTEC, Noordwijk, Netherlands

W.-H. Ip · Z.-Y. Li

National Central University, Taipei, Taiwan

E. Jessberger

University of Münster, Münster, Germany 
activity to the perihelion of comet CG will provide extensive experience on operating inside the coma. The risks of operations in the vicinity of a cometary nucleus will be fully understood and can be minimised during sampling. Adequate knowledge to provide samples from scientifically significant locations will be available.

Section 3 demonstrates that a sample from a short period (Jupiter-Family) comet can be returned by a spacecraft that is considerably smaller than Rosetta. The length of the mission is typically 10 years. A spacecraft launched in April 2018 by a Soyuz launcher to comet 79P/du Toit-Hartley will return $1.5 \mathrm{~kg}$ of cooled cometary material in April 2028. We chose a conservative approach using systems relying on technologies that are either existing or to be developed for approved missions like BepiColombo. The same holds true for the trajectory calculations of the Solar-Electric Propulsion (SEP) driven spacecraft. The Triple $F$ mission is proposed in collaboration with ROSCOSMOS (Russian Space Agency) and the total budget is $600 \mathrm{M} €$, equally shared between ESA and ROSCOSMOS.

\section{Scientific goals of a CNSR mission}

2.1 Relevance of comets for solar system formation

The scientific questions to be addressed in this mission have been raised by past space missions to comets, particularly to Halley (Giotto, Vega), Tempel 1

G. Kargl • N. I. Kömle

IWF, Graz, Austria

K. Kossacki

University of Warsaw, Warsaw, Poland

J. Licandro

IAC, Tenerife, Spain

S. C. Lowry

University of Belfast, Belfast, UK

I. Mann

University of Kobe, Kobe, Japan

Z. Martins · M. Schönbächler Imperial College, London, UK

M. Maurette

CNRS, Orsay, France

U. Meierhenrich

University of Nice, Nice, France

L. R. Nittler

Carnegie Institution, Washington, USA

亚 Springer 
(Deep Impact) and Wild 2 (Stardust), but also by observations made with space telescopes (HST, ISO, SST), and extensive ground-based observing campaigns. What is the complement of pristine interstellar organic material in comets? Is it possible to reconstruct the physical and chemical history of interstellar material in the nebula from observations of its state in comets? Does the chemical composition of a comet reflect its formation zone in the nebula? Do different cometary materials (dust, ices, organic compounds) originate from markedly different environments? If so, what are its implications for the physical conditions (temperature, density) of nebular evolution? The scientific returns from this mission will allow these questions to be answered and permit fundamental progress to be made in advancing knowledge of the origin of our Solar System.

\title{
2.1.1 Formation of the solar system from the protosolar cloud
}

About 4.6 billion years ago the Solar Nebula formed from a collapsing fragment of a molecular cloud (MC). Isotopic evidence associates the collapse with explosive injection of possibly at least two pulses of material from a nearby supernova [7]. In the first phase of this process, which lasted about $10^{5}$ years, a protostar with a surrounding thick disk formed, deeply embedded in its parental MC. During the second phase this disk became thinner and reached a size of $100 \mathrm{AU}$ or more in a time of less than $3 \times 10^{6}$ years. At the end of this phase the solar nebula consisted of a viscous gas-dust mixture. Due to

\author{
M. Pätzold \\ University of Cologne, Cologne, Germany \\ S. Sandford \\ NASA Ames Research Center, Moffett Field, USA \\ R. M. Stroud \\ Naval Research Laboratory, Washington, USA \\ S. Szutowicz \\ Space Research Center of PAS, Warsaw, Poland \\ S. Ulamec \\ German Aerospace Center (DLR), Cologne, Germany \\ M. Wallis \\ University of Cardiff, Cardiff, UK \\ W. Waniak \\ University of Krakow, Krakow, Poland \\ P. Weissman \\ JPL, Pasadena, USA \\ R. Wieler \\ ETH, Zürich, Switzerland
}


the gas-dust interaction the coagulating grains settled to the mid-plane of the disk. Contemporaneously, the stellar accretion rate decreased and the protoSun reached its T Tauri state. Analogues of this ancient star forming process are observable in our galaxy today, e.g., in the Orion or Taurus Molecular Cloud. In contrast to the Taurus region, the star formation in the Orion MC is influenced by intense radiation in the far UV and by external shocks. Currently, we do not know whether our Sun has formed in a MC more similar to Orion or to Taurus. However, the different conditions prevailing during the formation of the solar system have left traces that can be found today in cometary material, such as the structure (amorphous or crystallized) and composition of the ice and dust fractions as well as pre-solar grains (identified by anomalous isotopes) and deuterated compounds or particular substances (e.g., PAHs). The Stardust mission recovered a few, probably pre-solar grains from comet Wild 2 [57], but the abundance of these grains in comets is as yet very uncertain. In the third phase, the dust grains decoupled from the gas, with the latter eventually being blown off from the disk. The solar nebula evolved to a planet-forming debris disk. This evolution took $(3-10) \times 10^{7}$ years and it is not yet fully understood. Planetesimals accreted to planets with fundamental difference inside and outside the snowline. The material that accreted beyond this snowline can be found in comets. The composition of comets, as well as the differences between comets and the different types of asteroids, provides constraints on the solar nebula evolution in phase three. As a prerequisite, one needs to distinguish between the influence of the evolving solar nebula on the forming comets and their potential subsequent processing.

\subsubsection{Comets as remnants of solar system formation}

As witnessed by the high abundance of volatile ices such as CO in cometary nuclei, comets formed and spent most of their lifetime in a cold environment. Due to their origin in the coldest part of the solar nebula, comets are the solar system objects that underwent the least processing since the formation of the solar system from the pre-solar cloud.

While it is evident that comets consist of the best preserved material from the solar nebula, it is a longstanding question to what extent material from short period comets evolved since its formation. New results, from the Deep Impact mission as well as from laboratory experiments and modeling efforts, suggest that we can rule out previous suggestions that cometary materials were highly processed to great depths below the surface.

An aging process operating on a short period comet is collisional processing in the Kuiper belt [19]. While such a process would hardly affect the chemical and mineralogical properties of the comet, it can be expected to change physical properties like strength and density [8]. Recent results suggest that the history of short period comets may be less violent than suggested previously. For example, it is suggested that the origin of many short period comets is the scattered disk, a collisionally much more benign environment than the classical Kuiper belt [17]. Also, Deep Impact has shown evidence for primordial 
layering in comet Tempel 1 [6], suggesting that at least this comet is not a collisional fragment.

After injection into the inner solar system the surface layers of a comet are processed by solar heating and sublimation of volatiles. Models of the evolution of cometary surface layers disagree about the depth of the surface layer that was processed by sunlight. Deep Impact measurements $[29,60]$ of Comet Tempel 1 suggest that the thermal inertia of the surface layer of a comet is so low that, in an active area, the timescale of the penetration of the solar heat wave is comparable to that of removal of surface material by sublimation. A sample of an active region obtained around or shortly after perihelion will therefore be largely unaltered by solar heating even near the surface.

A cometary sample provides the unique opportunity to return pristine material to Earth. After the return of a small sample of the refractory component of a comet by Stardust and the upcoming in-depth investigation of a cometary surface by Rosetta, the return of a sample of largely unprocessed primordial material is the logical next step.

\subsubsection{Potential of Triple $F$}

The Wild 2 material in the Stardust sample was predominantly fine dust from our solar nebula, and not preserved isotopically anomalous pre-solar material (e.g., based upon the oxygen isotopic compositions [42]). The dust was mostly crystalline, not amorphous, and at least $10 \%$ of these crystalline materials appear to have originated in the inner solar system, not in the region where the Kuiper belt objects were assembled. A cometary sample analyzed with the powerful techniques available on Earth will shed more light into the composition of a comet and the origin of its components. It would also contain much coarser-grained materials and ices and, contrary to the aerogel capture of the Stardust samples, the sampled material will suffer little modification. Therefore, it will provide a much more complete picture of the processes operating in the early solar system.

Kuiper belt comets carry unique information on materials and processes across the entire solar nebula disk. For this reason direct comparison with undifferentiated asteroids (formed in the inner part of the disk) is important. Kuiper belt comets will also provide new information on the first generation planetesimals that formed the primitive asteroids, since in the comets these primordial materials are packed in ice and not heated as they were in many asteroids. However, to extract such information large returned sample masses will be required (hundreds of grams). Stardust collected $<1 \mathrm{mg}$ of very fine, refractory dust and only traces of volatiles [41, 54]. Large sample masses will permit radiometric dating even of minor cometary components, which are inherited from many different bodies, including broken up large Kuiper belt objects (see [9]). Only large sample masses will permit detailed studies of minor, unaltered organic components, including amino acids, and will provide sufficiently large quantities of unaltered presolar grains for detailed studies of nucleosynthesis and processing in the insterstellar medium. 


\subsection{Comets and life on Earth}

In the endeavor to understand the different steps towards the origin of life on Earth and in a wider context in the Universe, one of the prerequisites is to identify the premises of emerging life. It is suggested that life emerged in water and that the first self-replicating molecules and their precursors were organic molecules of growing complexity. It is still an open question, however, whether the organic starting material relevant to the origin of life was produced in-situ on the primitive Earth or whether it was delivered from space. These two processes may not be exclusive but may rather represent complementary contributions towards the origin of life.

Comets represent the most accessible target for acquiring materials formed in the outer part of the solar system. How well has this material been preserved since the formation of our solar system? What is their inventory of complex organic molecules and what was their role in the processes leading to the emergence of life on Earth? Answers to these basic questions will also provide essential complementary information to the European science-driven aspects of the space exploration program with the overarching scientific goal to reach a better understanding of the emergence and co-evolution of life with its planetary environments. However, it must be stressed that the absence of liquid water in comets over long periods of time greatly diminishes, if not completely eliminates, the possibility of the existence of living organisms in or on comets.

\subsubsection{Organics and prebiotic molecules}

Comets probably contributed part of the carbonaceous compounds during the heavy bombardment phase in the inner solar system including the Earth 4.5-4 billion years ago [18]. Material arriving from outside may have been crucial for the evolution of carbon chemistry and subsequently life, since the atmosphere and surface of the early Earth were likely not favorable to organic syntheses. The terrestrial accretion process itself and the subsequent core differentiation as well as the impact events, are important energy sources which kept the surface of the early Earth fairly hot [40] and covered with volcanoes. Amino acids - the building blocks of proteins - and nucleic acids have been found in several carbonaceous chondrites. The small L-enantiomeric excess of amino acids measured in those meteorites indicates that the origin of asymmetric amino acid formation is not yet well understood.

Whereas the organic inventory of meteorites can be investigated in the laboratory by use of sophisticated analytical techniques, cometary nuclei-so far-evade our direct access. Our current knowledge is based on data from the different fly-by missions, and from the Stardust probe that brought back cometary grains from the coma of comet Wild 2. An organic component was identified in the Stardust samples which is richer in oxygen and nitrogen than organic compounds found in carbonaceous meteorites and Halley dust, indicating a different chemical composition and thus different chemical pathways 
to its formation [54]. Polycyclic aromatic hydrocarbons (PAHs) have been observed in the Stardust samples, such as naphthalene $\left(\mathrm{C}_{10} \mathrm{H}_{8}\right)$, phenanthrene $\left(\mathrm{C}_{14} \mathrm{H}_{10}\right)$ and pyrene $\left(\mathrm{C}_{16} \mathrm{H}_{10}\right)$. From laboratory simulation experiments, the existence of more complex molecular structures in comet nuclei is inferred. However, so far, we have not yet succeeded in a direct identification of complex organic molecules in cometary nuclei. One goal of the Triple $F$ mission is to study the ratio of simple to complex organic molecules in the cometary nucleus. For instance, so far it is not known [21] whether $\mathrm{H}_{2} \mathrm{CO}$ is an original nucleus molecule or rather a daughter molecule of POM (polyoxymethylene). The same is true for monomeric or polymeric HCN [25].

There is strong evidence that amorphous carbon and similar macromolecular material account for most of the carbon in the interstellar medium [43]. The same trend is observed in meteorites, where macromolecular material accounts for more than $80 \%$ of the carbon [23]. The link between macromolecular carbon in the solar system and the interstellar macromolecular carbon is yet to be understood, but it is tempting to assume that such a material is also present in comets. Apart from a major fraction of aromatic solid carbon, minor abundances of many organic molecules, probably including prebiotic ones, will be present in the cometary nucleus.

Living organisms are based on (a) left-handed amino acids that form proteins (biocatalysts, enzymes); and (b) nucleotide bases, phosphoric acid and right-handed ribose (sugar, carbohydrate) that form the genetic material DNA and RNA. The detection of life's precursor molecules in comets would provide important constraints for the origin of life on Earth and possibly elsewhere. The exact determination of enantiomeric ratios (and isotopic compositions) of prebiotic molecules in a sample returned from a comet will provide invaluable insights into the place and means of origin of the molecules important to the development of living systems.

\subsubsection{Hydrosphere and atmosphere}

Two sources of water on Earth are commonly envisioned: Adsorption of water by grains in the accretion disk [16] or delivery by comets and asteroids [15, 45]. The $\mathrm{D} / \mathrm{H}$ ratio of SMOW (Standard Mean Ocean Water) is only half the $\mathrm{D} / \mathrm{H}$ ratio of cometary water, and hence the suggestion that comets are a major source of terrestrial water is questionable. So far the cometary $\mathrm{D} / \mathrm{H}$ ratio is estimated from the coma of three long-period and Halley-type comets: Halley, Hale-Bopp, and Hyakutake. It is unclear if the ratio is the same in short-period comets. Since comets formed from components that were created over a wide range of heliocentric distances, the $\mathrm{D} / \mathrm{H}$ ratio may also vary between different ice crystals in the same comet, providing information about the variation of $\mathrm{D} / \mathrm{H}$ in the solar nebula. Therefore, we need to determine the $\mathrm{D} / \mathrm{H}$ ratio in various water ice aggregates and its variation within the comet.

A further question is related to the formation of the terrestrial atmosphere. Preferred gas components used to investigate this question are the isotopes of the noble gases and nitrogen. The former are chemically inert and the 
latter corresponds to the major part of the current atmosphere. We know that the composition of the terrestrial atmosphere is not solar. Consequently, any realistic formation concept has to consider an evolutionary process starting from a primordial atmosphere. The evolution itself is caused by [51]:

- gravitational escape, probably driven by the Moon-forming impact and by the adsorption of intense ultraviolet radiation from the young Sun and

- planetary degassing.

Various scenarios describe how Earth could have acquired its primordial atmosphere. The primordial atmosphere could have been captured gravitationally from the gas of the surrounding solar nebula [52], or the atmospheric volatiles resulted from gases adsorbed on the infalling planetesimals during the accretion phase. The abundances of $\mathrm{Ar}, \mathrm{Kr}$ and $\mathrm{Xe}$ on Mars, Earth and Venus suggest that comets could have delivered considerable amounts of these gases, along with other volatiles, to these planets at the end of the late bombardment period [49]. If one measures the elemental and isotopic noble gas ratios (e.g., ${ }^{4} \mathrm{He} /{ }^{20} \mathrm{Ne},{ }^{4} \mathrm{He} /{ }^{36} \mathrm{Ar},{ }^{20} \mathrm{Ne} /{ }^{22} \mathrm{Ne},{ }^{21} \mathrm{Ne} /{ }^{22} \mathrm{Ne},{ }^{136} \mathrm{Xe} /{ }^{130} \mathrm{Xe}$, ${ }^{129} \mathrm{Xe} /{ }^{130} \mathrm{Xe}$ ) and ${ }^{15} \mathrm{~N} /{ }^{14} \mathrm{~N}$ of the material provided by the Triple $F$ mission, the potential cometary source, and hence the various scenarios, can be evaluated.

\subsubsection{Potential of Triple F}

A cometary nucleus sample return mission will be a crucial step in the investigation of the organic component and isotopic ratios of a cometary nucleus. The investigation of large samples with sophisticated analytical instruments in specialized laboratories will allow us to study in detail the variety of organic compounds including both large and small organic molecules, complex carbonaceous material, the organic-mineral connections in comets, and isotopic ratios.

The Rosetta mission on the way to Comet 67P/Churyumov-Gerasimenko carries instruments that will study the in-situ chemical composition of the comet nucleus. However, Rosetta is limited in the analysis of complex organics. The instrument COSAC [28] on the Rosetta Lander Philae will methylize nonvolatile compounds such as carboxylic acids as well as amino acids to make them visible for gas chromatographic analyses. However, many of the larger organic molecules cannot be analyzed with Rosetta's in-situ instrumentation. One important analysis that is not covered is sugar chemistry. Amino acids and their polymers are possibly accessible by derivatisation and GC-MS, but sugars and their polymers need methods too sophisticated for the kind of space instrumentation used on Rosetta's lander Philae. Similarly, the analyses of isotopic ratios is restricted to light elements and limited by sample size.

\subsection{The mystery of cometary activity}

In spite of substantial observational, experimental, and theoretical efforts, cometary activity is far from being understood. However, knowing how activity 
works is vital for assessing how the pristine material from which comets formed has been processed and possibly altered over time. An answer may be found by combining data from the ESA mission Rosetta and the currently proposed mission. The latter will deliver complementary, unique, and extremely important information regarding a number of basic questions, discussed below. Some questions will be answered by conducting direct measurements on the retrieved material; others can be derived from these measurements in combination with modeling. However, there is no obvious way to settle the issue without bringing a sufficiently large and relatively unaltered sample of cometary material to Earth for analysis.

\subsubsection{Heterogeneous distribution of active areas across the surface}

The asymmetric shapes of the cometary gas production curves relative to perihelion as well as the asymmetric non-gravitational forces perturbing the cometary orbits are indirect evidence of discrete outgassing regions on the nuclei. More directly, the existence of jets, fans, shells and other structures in comae indicate an anisotropic emission of gases and possible "active areas" on the surface of the nucleus. The ESA Giotto mission to Comet 1P/Halley in 1986 provided the first close-up imaging of a cometary nucleus that turned out to have complex surface structures with dust emission restricted to a few "active regions" covering about $20 \%$ of the sunlit side of the nucleus [32, 33]. The close encounter with 19P/Borrelly (NASA Deep Space 1 mission, 2001) revealed narrow, highly collimated structures similar to those already seen in $1 \mathrm{P} /$ Halley [55]. Analysis of the surface morphology and albedo variations suggests that some landforms (e.g. mottled terrain) represent surface subjected to extensive sublimation-driven erosion in the past, while other features (e.g. bright-appearing slopes of mesas) are probably freshly exposed sources of some of the active jets [34]. Observations of Comet 9P/Tempel 1 by the NASA Deep Impact mission show differing coma distribution patterns of water and carbon dioxide with a high degree of spatial asymmetry [20]. Therefore, the mixture of active and inactive areas appears to be common, but the intrinsic differences between such surface types are unknown.

\subsubsection{Depth of the water sublimation front in active areas}

It is unclear if coma gas primarily originates from exposed surface ice, or from shallow sub-surface regions, but various arguments suggest that in an active area we find volatile material within a few $\mathrm{cm}$ of the surface. There certainly is water ice on the surface of Comet $9 \mathrm{P} /$ Tempel 1 , as shown by spectral absorptions at 1.5 and $2.0 \mu \mathrm{m}$ [59]. However, the estimated active area fraction [14] is at odds with the area fraction actually showing water absorption features. Furthermore, the distribution of water just above the surface strongly suggests that the bulk of the outgassing takes place along the noon meridian, which is on the visible side of the nucleus in a region that must have less than 
$1 \%$ surface ice coverage [20]. This implies subsurface sources, unless the far (unimaged) side of the nucleus is richer in ice, and/or additional (undetected) ice is present on the imaged side. If subsurface sources of ice indeed dominate cometary outgassing, chances are still excellent to find sublimation fronts well within the sampled $50 \mathrm{~cm}$ depth. Laboratory work [30] shows that a thin refractory mantle of some $\mathrm{cm}$ would strongly quench the outgassing, much below the level observed at comets. Both the analysis of the Hale-Bopp data [36] and the Deep Impact measurements of surface temperatures on Tempel 1 [29] show that the thermal conductivity of the surface material must be very low $(\leq 0.01 \mathrm{~W} / \mathrm{m} \mathrm{K})$. This also implies that potential dust mantles in active areas must be very thin ( $\mathrm{mm}$ to $\mathrm{cm}$ range), otherwise it would not be possible to transport enough heat to sublimate the water ice below [35].

An inactive area is probably characterized by a deeper refractory-volatile boundary. The absence of water absorption features in up range rays of Deep Impact ejecta and strong water ice absorption at $3.0 \mu \mathrm{m}$ in the remaining ejecta indicate a stratified surface at the most likely inactive impact site, with an icerich interior covered by an $\sim 1 \mathrm{~m}$ thick layer of dry material [60].

\subsubsection{Physical, chemical, structural, and mechanical properties of near-surface material on a microscopic scale}

The short- and long-term evolution of cometary material depends on its microphysical properties and the illumination conditions. To understand the outgassing processes and the physical properties that distinguish an active from an inactive region, thermal models must be supplied with physical, structural and mechanical parameters, such as heat conductivity, heat capacity, porosity, size distributions of grains and pores, pore connectivity, tensile strength, chemical composition et cetera. Such parameters change with surface location, depth, time, and in some cases, temperature. They can only be measured accurately and systematically in a sample of sufficient size in well-equipped laboratories. Such detailed information on microphysics is vital for explaining phenomena occurring on a global scale, over extended periods of time.

\subsubsection{Conditions for dust mantle formation}

Activity of comets at the same heliocentric distance of succeeding orbits is relatively constant. However, laboratory work on comet analog material has shown that ice and dust mixtures irradiated by solar light are quickly depleted in their water content at the surface by forming an insulating dust mantle, and activity drops quickly. Even taking into consideration that the presence of Earth's much higher gravity may cause results not directly applicable to comets, it remains true that the gas pressure of the volatiles is much lower than the Van der Waals forces between particles [37]. How then is activity maintained over time? 


\subsubsection{Intrinsic exothermal processes}

Cometary outbursts and distant activity are common phenomena. Exothermic reactions, such as crystallization of amorphous ice [5, 38], are perhaps the strongest candidate for delivering energy for driving outbursts and dust blowoff. While it is difficult to avoid crystallization of amorphous ice during the transport from the comet to Earth, amorphous ice may be detected indirectly by a temperature increase in the sample during crystallization.

\subsubsection{Potential of Triple $F$}

Rosetta with its 21 experiments will provide important input to solve the questions listed above by investigations of Comet 67P/Churyumov-Gerasimenko. However, there are some limitations that will be overcome by the proposed mission:

- Laboratory measurements of microphysical parameters can be made in substantially larger detail and more systematically than in situ.

- The Lander Philae will go down to only one (probably inactive) area. Triple $F$ will visit several regions with different morphologies and outgassing levels, exploring the reasons for diversity.

- The size of samples investigated with Philae $\left(10\right.$ to $\left.40 \mathrm{~mm}^{3}\right)$ is limited. The volume of samples returned will be orders of magnitudes larger and the samples will cover a wider depth range.

- The sample analysis is not limited by the technology and resources at the time the spacecraft is developed-analyses can be made using the full capability of terrestrial laboratories and new analyses can be made of curated samples as questions and techniques evolve.

\section{The mission concept}

\subsection{Overview}

The baseline mission foresees a launch in April 2018. The spacecraft driven by Solar-Electric Propulsion (SEP) will fly-by Earth in 2019 and arrive at the target comet 79P/du Toit-Hartley in mid-2023. During approximately 6 months of operations at the comet the spacecraft will get samples from three surface locations of the cometary nucleus by touch-and-go sampling. To prepare sampling and to enhance the scientific return, the comet will be investigated by remote sensing instruments and in situ dust and gas measurements. The sampling devices are corers that will be driven into the cometary surface once the spacecraft touches the ground. Ground contact will be for a few seconds only. After retrieving the samples, return travel to Earth will begin in early 2024. The samples are cooled down to $133 \mathrm{~K}$ during the complete return travel, except for $2 \mathrm{~h}$ at re-entry into Earth's atmosphere when the temperature will 
increase to $163 \mathrm{~K}$. The spacecraft will return to Earth and deliver the re-entry capsule in April 2028, after a total mission duration of 10 years.

\subsection{The target comet: 79P/duToit-Hartley}

A mission to Comet 79P/du Toit-Hartley (hereafter 79P) was selected as the baseline because of the relatively low $\Delta V$ of approximately $10.3 \mathrm{~km} / \mathrm{s}$ required for the round trip and because it offers a launch opportunity at the beginning of the Cosmic Vision timeframe. Five other comets with $\Delta V$ between $10.0 \mathrm{~km} / \mathrm{s}$ and $11.3 \mathrm{~km} / \mathrm{s}$ were identified.

Comet 79P was detected by D. du Toit from South Africa in 1945. After its first observed perihelion passage it was lost for several decades. Finally it was recovered by M. Hartley in 1982, after a probable splitting event in 1976. 79P was observed again by professional astronomers during its last two orbits in 1995 and 2003. From observations of its inactive nucleus at large distance from the Sun the radius of 79P is estimated to be $1.4 \pm 0.3 \mathrm{~km}$ [39].

The perihelion distance of $79 \mathrm{P}$ is currently $1.23 \mathrm{AU}$, but it will decrease to 1.12 AU by its perihelion in 2023 when it will be visited by Triple F. Its aphelion distance of $4.8 \mathrm{AU}$ and orbital inclination of $3^{\circ}$ are both relatively low, making $79 \mathrm{P}$ a good target for a sample return mission.

\subsection{Launcher}

For the Triple $F$ mission a Soyuz launch from Kourou is considered. For Soyuz both, direct escape and escape from Geostationary Transfer Orbit (GTO) using a propulsion module are attractive. Various escape scenarios are possible. As the baseline, we consider use of a propulsion module and a lunar gravity assist which results in an escape mass of 2,000 $\mathrm{kg}$ and an excess velocity at escape of $1,100 \mathrm{~m} / \mathrm{s}$.

\subsection{The interplanetary trajectory}

The baseline trajectory for the Triple F mission is outlined in Fig. 1. An optimal solution was found for a launch date in April 2018. The round trip mission duration for this option is 10 years. The stay time at the comet is 7 months which provides sufficient time for a characterization of the comet and the sampling.

\subsubsection{Transfer}

Even with a state of the art electric propulsion system a direct transfer to Jupiter-family comets such as $79 / \mathrm{P}$ is not possible within reasonable time. A generically applicable strategy to reduce the $\Delta V$ for the transfer to a comet is 
Fig. 1 Transfer (a) and return (b) trajectories to comet 79P/du Toit-Hartley (dimensions in AU).

Thrusting periods are shown in red

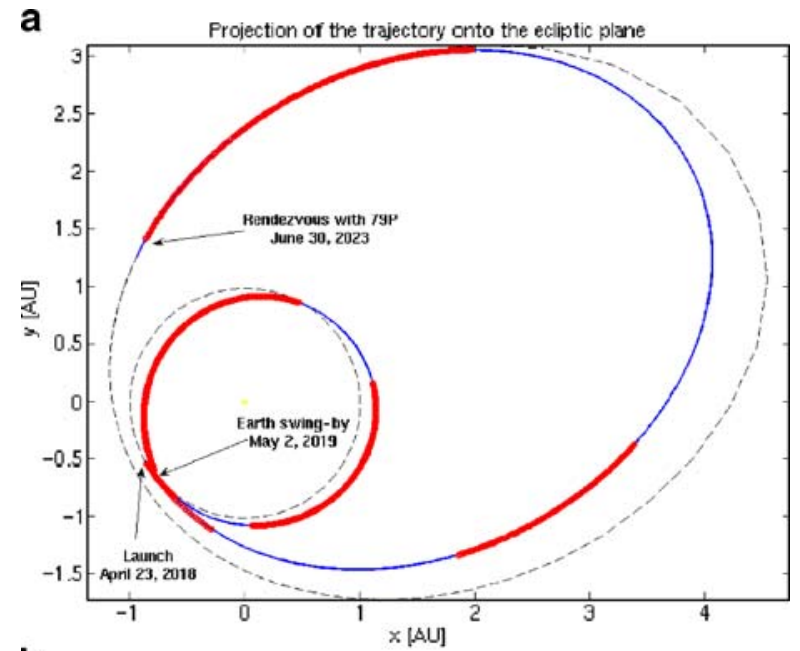

b

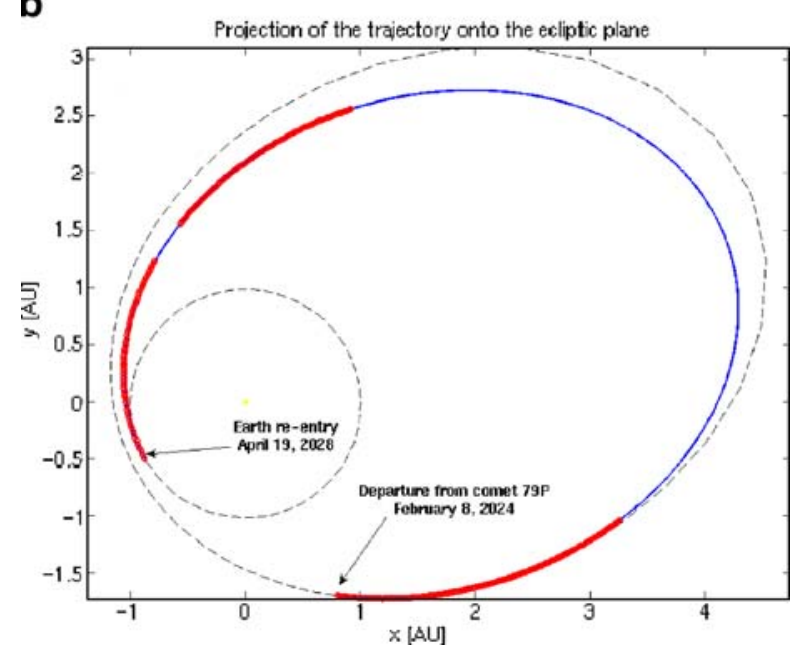

the return to Earth for a gravity assist. The basic sequence for the transfer to the comet is the following:

- Launch into GTO

- Raise apogee to lunar crossing orbit by chemical propulsion stage (requires $\sim 700 \mathrm{~m} / \mathrm{s}$ from GTO)

- Perform a Lunar Gravity Assist

- Leave Earth and thrust for increasing eccentricity of heliocentric orbit and for targeting Earth

- Come back to Earth after little more than 1 year and perform an Earth Gravity Assist. 
For the return trajectory it needs to be taken into account that the re-entry velocity into the Earths atmosphere shall not exceed a certain value. For the proposal a maximum reentry velocity of $13 \mathrm{~km} / \mathrm{s}$ is chosen that lies within the range that was already demonstrated by previous missions (e.g. Stardust, Genesis). Hence the re-entry velocity is limited to $13 \mathrm{~km} / \mathrm{s}$. In order to comply with this limit, the return trajectory requires a braking maneuver that adds to the roundtrip $\Delta V$.

\subsubsection{Comet approach}

Due to the low-thrust transfer the spacecraft will approach the comet at a very low relative velocity. The rendezvous will be achieved by a series of small maneuvers. In order to ensure collision avoidance during this phase (with the comet's gravity field still badly known), the maneuvers will never directly target the comet but always some point outside of its cross section. This strategy is also foreseen for Rosetta. However, the rendezvous maneuver sequence for Triple $F$ is less risky due to the much lower relative velocity.

\subsubsection{Re-entry}

For the re-entry the Triple $F$ spacecraft targets a hyperbola with its perigee at the Earth's surface. Approximately $5 \mathrm{~h}$ before the perigee the re-entry capsule is deployed.

The re-entry capsule will follow the desired re-entry trajectory entirely passively and without maneuver capability. After the deployment of the reentry capsule the spacecraft conducts an orbit maneuver that puts itself on an Earth fly-by trajectory in order to avoid destruction.

\subsection{Spacecraft}

The basic concept of Triple F is simple: A single spacecraft will be sent to the comet, acquire the sample, and return. Due to the low gravity environment, the propellant penalty for this concept compared to a mothercraft-lander system is minimal and one can avoid the complexity of having to rendezvous the mothercraft with the lander in order to transfer the sample. The preferred sampling concept is that of a touch-and-go sampling. The whole maneuver will typically take about $1 \mathrm{~h}$. Limiting factors are the rotational period of the comet and the need that the whole approach sampling and take-off sequence has to be carried out in daylight. The conclusion that touch-and-go sampling with the whole spacecraft is the preferred operational scenario had already been reached for the Hayabusa asteroid sample return mission [22] and also during the Asteroid Sample Return Technology Reference Study [1] for ESA. A major advantage is that the thermal control of the spacecraft does not have to be adjusted to the "hot" surface and that all resources of the main spacecraft are available. 
Fig. 2 Triple $F$ spacecraft configuration concept

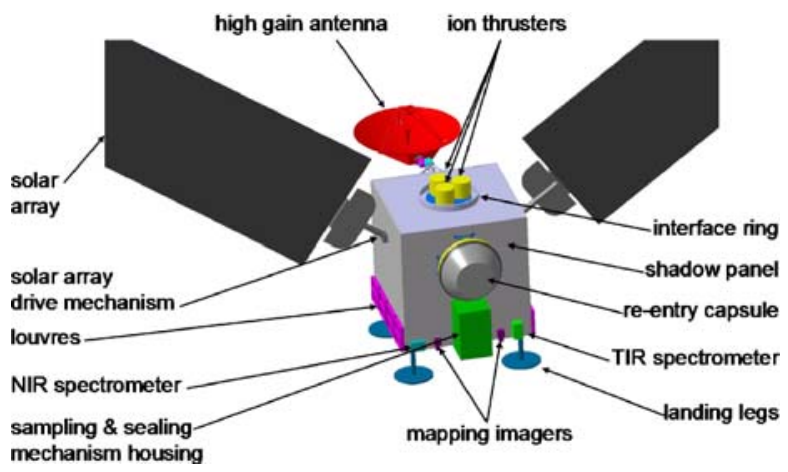

A major challenge is to reconcile the large wingspan of the solar arrays of approximately $15 \mathrm{~m}$ each, that is required by the electric propulsion system, with the need to avoid simultaneous ground contact of both solar panels (with the spacecraft continuing to move downwards) when landing. Our concept uses a 1 degrees-of-freedom (DOF) rotational mechanism so that when moving, the solar array follows an imaginary cone. To maximize ground clearance, the solar array will have to be turned in the morning/evening orientation when landing which is compatible with the desired landing scenario. The option yields optimal ground clearance and makes use of an existing solar array drive mechanism. The disadvantage of the concept is the motion of the centre of mass of the spacecraft introduced by the solar panel movement. However, this appears not critical, since it will be compensated by the thruster pointing mechanism of the electric propulsion system.

This thruster pointing mechanism is mandatory anyway in order to minimize the angular momentum build-up that would otherwise result from shifts in the spacecraft's center of mass due to fuel consumption and antenna pointing. Landing legs are foreseen to guarantee clearance of the attitude control thrusters from the ground.

A 2 DOF articulated high gain antenna (HGA) is proposed to ensure permanent radio link to the spacecraft during thrust phases and to allow simultaneous remote observations and data downlink at the comet.

The re-entry capsule needs to be placed on a panel of the spacecraft that is permanently in shadow. This panel also accommodates the sampling mechanism. The resulting spacecraft configuration is shown in Fig. 2.

\subsubsection{Solar electric propulsion}

The mission is based on the simultaneous operation of two RIT22 or T6 thrusters. The total thrust time of nearly $20,000 \mathrm{~h}$ per thruster is well within the $25,000 \mathrm{~h}$ expected lifetime capability of the BepiColombo thruster. In addition a third thruster is foreseen for redundancy. The architecture of the propulsion system corresponds to that of BepiColombo hence extensive use of the BepiColombo heritage could be made. 


\subsubsection{Power, telecommunications and data handling}

For the Triple $F$ mission a solar array with an area of $60 \mathrm{~m}^{2}$ is foreseen. Considering aging and losses in the power processing units this corresponds to an available power of approximately $12,650 \mathrm{~W}$ at $1 \mathrm{AU}$. Of this power typically $500 \mathrm{~W}$ (+100 W margin) will be required for the bus leaving approximately $12,050 \mathrm{~W}$ to the electric propulsion system enabling a thrust level of $350 \mathrm{mN}$ at $1 \mathrm{AU}$. The available power will decay with heliocentric distance, $R$, roughly as $R^{-1.7}$ due to the improved solar cell efficiency at lower temperatures. Ample power is available during coast phases of the transfer and during the comet proximity operations. In particular, an off-pointing of the solar arrays from the Sun direction that may be necessary to achieve sufficient ground clearance of the solar array during the touch-and-go operations is fully compatible with the available power. The minimal thrust level of the RIT22 and T6 ion engines of $30 \mathrm{mN}$ is reached at $3.2 \mathrm{AU}$ heliocentric distance. Beyond this distance the electric propulsion system needs to be switched off.

The solar array sizing is entirely driven by the power demand of the electric propulsion system. Also the power system design is determined by the requirements of the electric propulsion system: A $50 \mathrm{~V}$ bus is needed for the propulsion system while the rest of the spacecraft is powered by a $28 \mathrm{~V}$ bus derived from the $50 \mathrm{~V}$ bus. The power system architecture can be based on the concept of BepiColombo.

An X-band telecommunication system based on BepiColombo with a transmit power of $27.5 \mathrm{~W}$ is foreseen for telemetry, tracking and telecommand and science data downlink. An additional Ka-band link for Doppler measurements is proposed in order to allow a precise determination of the comet's gravity field even at low angles between the Earth and the Sun as seen from the spacecraft. For the HGA a design similar to that of Mars Express and Rosetta with a diameter of $1.6 \mathrm{~m}$ is foreseen. This system will provide a data rate of 2 kbps over a distance of 5 AU.

The two drivers for the data handling system are on the one hand the Guidance, Navigation and Control (GNC) during the touch-and-go sequence and on the other hand the demands of the remote sensing instruments, e.g. data compression. For the GNC the requirements are reduced by the fact that the gravitational acceleration due to the comet is small, and hence, the approach will be slow. Consequently processors like the LEON III that will be available in the Cosmic Vision timeframe will be more than sufficient for the GNC. Probably even a current ERC32 with 14 Mips would fulfill the requirements. The requirements for the handling of the instrument data will depend on the processing capabilities that are already incorporated within the instruments.

\subsubsection{Guidance, navigation and control}

Three-axis stabilisation is the only viable control concept in order to fulfill the pointing needs during thrust phases and proximity operations. The attitude 
control system consists of sensors and actuators. A sun sensor is foreseen for initial attitude acquisition after launch and safe mode. Star trackers and an inertial measurement unit are used for attitude determination. Standard reaction wheels of $12 \mathrm{Nms}$ capacity and a set of 12 (+12 redundant) $10 \mathrm{~N}$ thrusters are foreseen as actuators. In all nominal modes the attitude control of Triple $F$ relies on reaction wheels. For safe mode the attitude control will rely on the thrusters. The thrusters are also used for wheel desaturation and orbit control.

The major challenge for the guidance navigation and control (GNC) are the comet proximity operations, including the touch-and-go on the comet. Years before the launch of the Triple $F$ mission, extended experience of operating in the near nucleus cometary environment will have been accumulated by Rosetta.

The rendezvous with the comet takes place at heliocentric distances below 2 AU. Hence, the spacecraft will be confronted with significant outgassing from the comet during its proximity operations. Due to the gas and dust flux from the comet, stable orbits may not exist and a different strategy must be devised. Attractive options are hovering, which was the nominal mode of Hayabusa, or an eclipse-free terminator orbit (low cometary activity), the latter being the baseline in Agnolon [1]. For the terminator orbit, regular eccentricity control must be conducted in order to compensate disturbances by the cometary environment. These correction maneuvers will have to rely on optical navigation based on landmark tracking or limb recognition. Due to the high operational effort of a ground controlled GNC during proximity operations, the implementation of autonomous GNC seems preferable. Also for the hovering strategy, a certain level of autonomous GNC for the spacecraft is required in order to avoid a strong deviation from the nominal position due to changes in the cometary environment.

Hazards due to the activity of the comet need to be considered. The gas streaming from the nucleus exerts a pressure on the solar panels that pushes the spacecraft away from the comet. However, even for active Jupiter-family comets the average gas pressure at heliocentric distances larger than $1 \mathrm{AU}$ is of the order of the gravity attraction. When the samples will be collected at a heliocentric distance of about $2 \mathrm{AU}$ it will be considerably less. This can easily be compensated by the SEP. The second hazard is fine dust leaving the nucleus; the importance of that hazard depends on the sticking properties of the dust particles and the ill-constrained dust size distribution. Our strategy to avoid high dust fluxes on the solar panels is twofold. Firstly, the solar panels will be directed in a way that the area pointing towards the comet is minimized (orbit near terminator). Secondly, when operating close to the comet, times (local noon) and regions of high dust activity will be avoided. The dust flux will be continuously monitored by the cameras and the in-situ dust instrument. Due to the experience from Rosetta we will have a good understanding of the expected dust environment and the knowledge how to operate close to a comet. There is ample time ( 7 months) to characterize the inhomogeneous activity of $79 \mathrm{P}$ and to devise a safe strategy before touch down. 
Ballistic descent from a low orbit is our baseline strategy for the GNC during the touch and go, following the conclusion of Agnolon [1]. He proposes to have the comet detection as well as the approach and orbit capture controlled by the ground, using radio navigation. The spacecraft would autonomously control the orbit and perform the landing and ascent maneuver, based on visual navigation with wide angle cameras. The duration of descent is estimated to range between 30 and $90 \mathrm{~min}$, the landing precision on the order of $10 \mathrm{~m}$.

The only major difference between the study by Agnolon [1] and Triple F as proposed here is the much larger size of the solar array due to the electric propulsion system. Avoiding the danger of ground contact with the solar arrays has already been discussed above. With this issue under control the GNC of Triple $F$ can be based on the findings of Agnolon [1] and does not pose challenges beyond those identified there.

\subsubsection{Thermal control}

The Rosetta spacecraft is currently demonstrating the thermal control of a spacecraft over a range of heliocentric distances from 1 to approximately $6 \mathrm{AU}$. The thermal control of Rosetta is achieved by louvers which open at high temperatures and close at low temperatures. This simple concept is also applicable to the Triple $F$ mission. More refined concepts could allow mass savings in the thermal control subsystems if required. In particular the louvers could be replaced by radiators and heat switches, which are currently being developed for the Exomars Rover in ESA's Aurora Programme.

A particular challenge of the Triple $F$ thermal control is to ensure a cryogenic temperature of the sample. In Section 3.6.1 it is argued that a temperature of at most $135 \mathrm{~K}$ during transfer and $170 \mathrm{~K}$ for the reentry is desirable to maximize the science return. We designed a simple thermal control that will perform reliably during the 4 years return transfer and keeps the temperature below $133 \mathrm{~K}$ in interplanetary space and $163 \mathrm{~K}$ during re-entry.

The sample container will be transferred to the reentry capsule soon after sample acquisition. The re-entry capsule will be kept in the shadow of the spacecraft during the complete return transfer. The capsule will have three zones at different temperatures which are well thermally decoupled from each other.

The desired temperature of the sample container can be maintained with a modest radiator size of $0.4 \times 0.4 \mathrm{~m}^{2}$ and a conductivity coefficient between the cold and the intermediate zone of $0.03 \mathrm{~W} / \mathrm{K}$. This is challenging due to the requirement to have a safe mechanical interface between the zones, but well within reach.

After the detachment of the re-entry capsule from the spacecraft the cooling of the sample container can no longer rely on the radiator of the return capsule because it may be exposed to sunlight due to the approach trajectory of the capsule towards Earth. Hence, during the final approach, the reentry and on ground before recovery of the capsule the sample container will be cooled from 
the heat capacitor to which it is coupled. This concept is feasible because the temperature on the backside of the ablative heat shield will be quite modest. For a preliminary assessment a conservative value of $523 \mathrm{~K}$ was assumed taking into account the values for Stardust. Allowing for a temperature rise of the sample container of $30 \mathrm{~K}$ to $163 \mathrm{~K}$ and assuming a re-entry duration of $15 \mathrm{~min}$ ( $14 \mathrm{~min}$ for Stardust), followed by $2 \mathrm{~h}$ on ground before recovery, a heat capacitor of $5 \mathrm{~kg}$ water and a conductivity coefficient of $0.26 \mathrm{~W} / \mathrm{K}$ between the backside of the heat shield and the cold zone is sufficient. Hence, the cooling of the sample during reentry is well compatible with the structural requirement of the re-entry capsule.

\subsubsection{Re-entry capsule}

There is a trade-off between the re-entry velocity of the return capsule and the braking $\Delta V$ of the Triple $F$ spacecraft on its return transfer. We have taken a conservative approach in limiting the entry velocity to $13 \mathrm{~km} / \mathrm{s}$. A detailed analysis may well reveal that a higher re-entry velocity is feasible and more mass efficient and could hence lead to a better overall system performance. In any case an ablative heat shield is considered mandatory. It is the most lightweight option and it facilitates the cooling of the sample because it leads to a rather low temperature of the backside of the heat shield. For the final stage of the descent a parachute is foreseen in order to avoid high mechanical loads on the sample at touchdown.

\subsubsection{Operations concept}

The mission operations concept for Triple $F$ shall ensure that the long mission duration of 10 years does not become a driver for the operational cost. Hence, a minimal number of ground contacts and a high level of spacecraft autonomy during transfer are desirable. For the mission the operational experience of the BepiColombo electric propulsion system will be available and hence it is assumed that also during thrust phase the spacecraft operations can be largely autonomous and ground contact can be reduced to once every week.

During the coast arcs of the transfer, ground contact will be infrequenttypically once per fortnight-because no specific telecommand or navigation needs arise during this phase. For the period around the Earth gravity assist and before the Earth re-entry permanent ground coverage and the use of delta-differential one-way ranging is desirable to achieve the best possible targeting accuracy.

For the comet rendezvous one ground station is sufficient and no large baseline tracking techniques are required. This low level of operational activity is possible due to the low approach velocity relative to the comet.

During the comet proximity operations the use of two or three ground stations is desirable in order to maximize the science return of this phase. However, the GNC strategy will be used to ensure safe operations of the 
spacecraft when no ground contact is possible and hence a permanent operational attention during the comet proximity phase is not mandatory.

The mission will use ESA $15 \mathrm{~m}$ ground stations for launch and early orbit operations as well as for apogee raising sequence for escape from GTO. ESA $35 \mathrm{~m}$ ground stations will be used for deep space communications during all other phases of the mission.

\subsection{Sampling, storage and analysis of samples}

\subsubsection{Requirements on sampling}

Number of samples and sampling locations on the comet The purpose of the mission is to return at least three samples from different locations on the surface of the comet. Samples will be taken from places with different levels of activity and at different "geographical" locations (with respect to the spin axis and, therefore, insolation), as surveyed by the payload instruments. Highest priority will be given to a sample from an area where ice is visible, or where activity has been observed. Then, an inactive area will be sampled, to compare active versus inactive regions. Finally, one sample will be taken from a (polar) region that sees little sunlight and therefore has experienced relatively little heating since the comet reached the inner solar system. Once the topography of the comet is known from the monitoring phase, additional criteria for the choice of the sampling site may become important. For example, it would be interesting to have a sample from a smooth area similar to the ones imaged on Tempel 1 [61], because there is the possibility that this material could have erupted from the subsurface.

Dimensions of the sample container As discussed in Section 2.3, a sample depth of $50 \mathrm{~cm}$ will be sufficient to find water ice in an active region. The diameter of the sample container is driven by the desired sample mass and by the requirement that sampling change the sample properties as little as possible. Determination of the formation history of the comet by radiometric dating requires several hundred grams of material (Section 2.1). For a density of $500 \mathrm{~kg} / \mathrm{m}^{3}$, a cylinder with a length of $50 \mathrm{~cm}$ and a diameter of $5 \mathrm{~cm}$ can collect $500 \mathrm{~g}$ of cometary material.

Any volatiles (including organic and inorganic molecules) that are present in the subsurface should be sampled in such a way as to avoid structural or compositional changes. Therefore, non-destructive sampling is important for the further analysis of the cometary material. The maximum allowed stress during sampling must not exceed the tensile, compressive, and shear strength of most of the sample. As we expect the cometary surface material to be extremely fragile, care must be taken to ensure that the sampler will not compress or otherwise alter the sample. We consider a sampling tube with a thin wall as the best non-invasive means to fulfill this criterion. Figure 3 shows an x-ray image of a cylindrical specimen of high-porosity ( $85 \%$ porosity) nonvolatile cometary analogue [8] which was sampled from a $2.5 \mathrm{~cm}$ diameter body 
Fig. 3 X-ray image of a sample taken from a $2.5 \mathrm{~cm}$ diameter high-porosity dust agglomerate by means of a tube sampler. The diameter of the sample is $7.5 \mathrm{~mm}$. Although the compressive strength of the sample is as low as $500 \mathrm{~Pa}$, the sample structure is preserved at distances $\geq 1 \mathrm{~mm}$ from the sampler wall

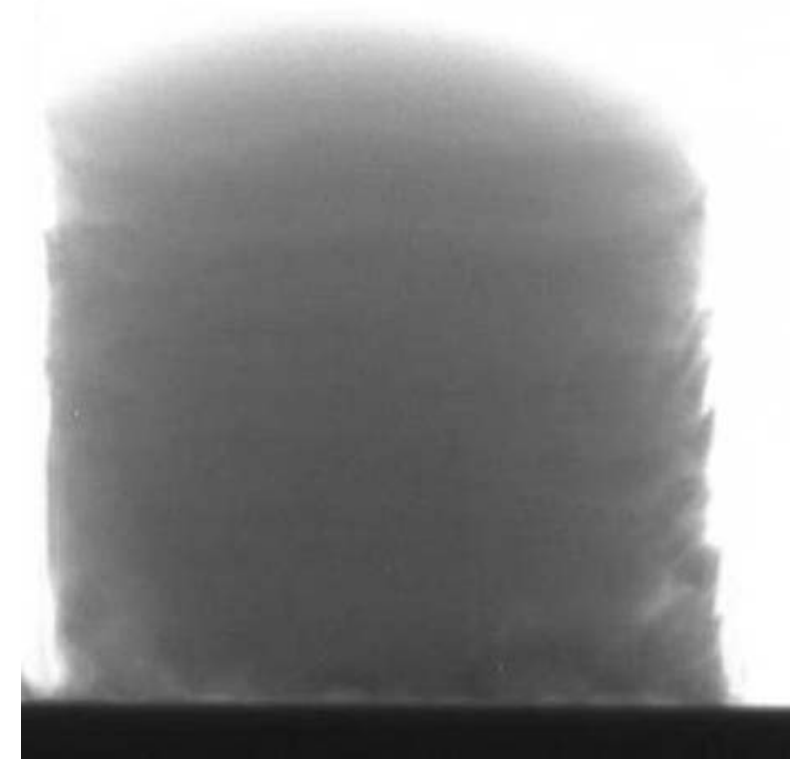

by means of a plastic tube (inner diameter $7.5 \mathrm{~mm}$, wall thickness $0.25 \mathrm{~mm}$ ). Although the outer edge shows a sawtooth shape, which stems from the manual operation of the sampler, the overall morphology of the sample is unaltered. In particular, the porosity of the sample is unchanged at distances $\geq 1 \mathrm{~mm}$ from the sampler wall. The sample itself is extremely fragile with a compressive strength of $500 \mathrm{~Pa}$ and a tensile strength of 1,000 Pa. For a sample diameter of $5 \mathrm{~cm}$, only a small fraction of the sample will be altered by interaction with the walls of the sampling tool.

Temperature of the sample during return to Earth The preservation of the sample micro-structure during sampling, cruising phase, re-entry into Earth's atmosphere and landing is vitally important for part of the mission science. The storage temperature of the samples is driven by the requirement that (at least) the most abundant volatile species, i.e. water ice, will be preserved in solid form. The relation between the gas mass and the initial solid phase mass as a function of the reservoir temperature $T_{\mathrm{R}}$ is:

$$
m / M_{\text {ice }}=\Psi /(\Psi-1) *\left(P_{\mathrm{V}} m_{\mathrm{g}}\right) /\left(\rho_{\text {ice }} k_{\mathrm{B}} T_{\mathrm{R}}\right)
$$

In the above equation $\Psi$ is the fraction of the probe volume filled by solid material, $P_{\mathrm{V}}$ is the vapour pressure, $m_{\mathrm{g}}$ the mass of a water molecule, and $\rho_{\text {ice }}$ the density of the ice. We used the Goff Gratch equation (Smithsonian Met. Tables, 5th ed., pp. 350,1984) to calculate $P_{\mathrm{V}}(T)$. The calculations show that up to a temperature of $190 \mathrm{~K}$ and a porosity of 0.7 , the fraction $\mathrm{m} / \mathrm{M}_{\text {ice }}$ is always below $2 * 10^{-7}$. A temperature $T \leq 200 \mathrm{~K}$ will guarantee that the ice 
will stay in cubic form (if cubic ice is present close to a cometary surface) and that sintering will be inefficient in solidifying ice-ice contacts.

The equilibrium calculation shows that during the whole return trip only a negligible fraction of the water ice will be in gaseous form. However, equilibrium is maintained by a large number of sublimations and recondensations. In the porous cometary material the gas molecules may move before recondensation, resulting in structural changes. As a worst case scenario, we assume that sublimation and subsequent recondensation always cause modification to the sample. In this case, the requirement is that the sublimation timescale (into vacuum) is larger than the travel time from the comet to Earth.

Figure 4 shows that a temperature of $135 \mathrm{~K}$ is sufficient to preserve the ice quantitatively (at the $90 \%$ level) in solid form over a mission time of 5 years. A temperature increase to $170 \mathrm{~K}$ during $2 \mathrm{~h}$ of atmospheric re-entry is acceptable.

The sample temperatures fulfill the requirement of preservation of the micro-structure of the sample. This does not necessarily mean (and it is not required for the scientific goals of the mission) that the sample remains completely unchanged. For example, due to the much higher vapour pressures of other cometary volatiles, a quantitative retention of minor ice species in solid form may require still lower temperatures. Also, should amorphous ice be present in the samples, the temperatures may not be sufficiently low to avoid its transformation into crystalline ice. However, maintaining substantially lower temperatures would be technically challenging. Therefore, we decided to identify volatile ices and amorphous water ice without trying to maintain them in their original state. Since the sampling device is sealed, a massspectroscopic analysis of the gas composition will unambiguously determine the total contents of all volatile species. Thermal probes on the sampling device will detect the temperature increase associated with the exothermal transformation of amorphous ice into crystalline ice.

Strength of the sampled material Unfortunately neither Deep Impact nor other comet observations measured the strength of cometary material. Various

Fig. 4 The percentage of sublimated water ice molecules is shown for a time span of 5 years (red curve) and of $2 \mathrm{~h}$ (blue curve)

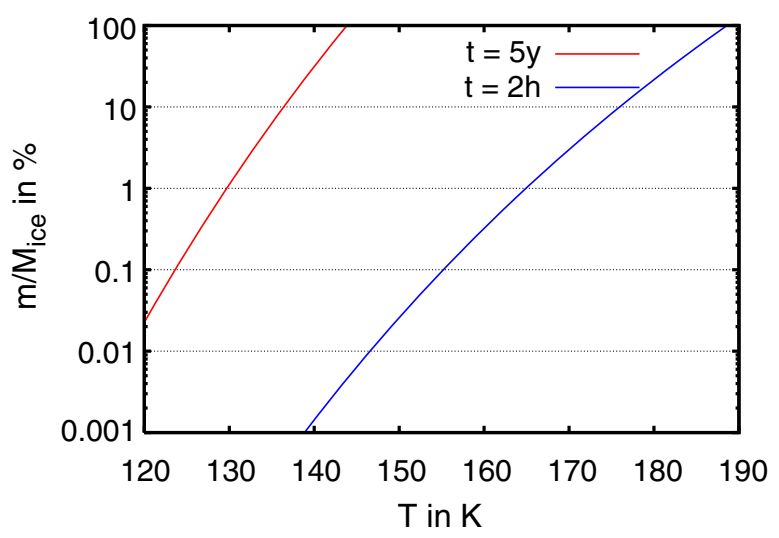


analyses of the impact cloud created by Deep Impact resulted in different upper limits for the strength of Comet Tempel 1 on scales of meters. The highest upper limit is $65 \mathrm{kPa}$ [31]. The few observational constraints we have for comets and cometary meteoroids as well as theoretical considerations and laboratory measurements [8] for weakly bound aggregates lead us to estimate the quasi-static tensile (or shear) strength of cometary material in the $\mathrm{dm}$ to m-range to be on the order of $1-10 \mathrm{kPa}$, while the compressive strength is estimated to be on the order of $10-100 \mathrm{kPa}$. We require the sampling mechanism to be able to sample materials with strength of up to $1 \mathrm{MPa}$. This corresponds to the highest values measured for sintered water ice.

\subsubsection{Concept of the sampling mechanism}

Several methods of sampling material from a small body have been discussed in Agnolon [1]. Here we show the preliminary design of a simple mechanism that allows rapid touch-and-go sampling (duration $\leq 2 \mathrm{~s}$ ) for the expected conditions of low or moderate surface strength of a comet. It was developed at the Polytechnic University of Hong Kong based on heritage from Beagle 2 [47].

A sampling mechanism with three corers is foreseen that can be activated separately in three different regions of the nucleus (Fig. 5). The device will use a tubular coring system with a length of $50 \mathrm{~cm}$ and a diameter of $50 \mathrm{~mm}$ that is propelled by a spring mechanism into the cometary surface. Since the bottom of the sampling cylinder is open during sampling, a shutter mechanism is needed that keeps the sample in the corer during its transport from the cometary surface to the spacecraft.

The acquired sample is directly delivered into a cooled transport container inside the re-entry capsule. Cross-contamination can be avoided by

Fig. 5 Global architecture of the sampling mechanism

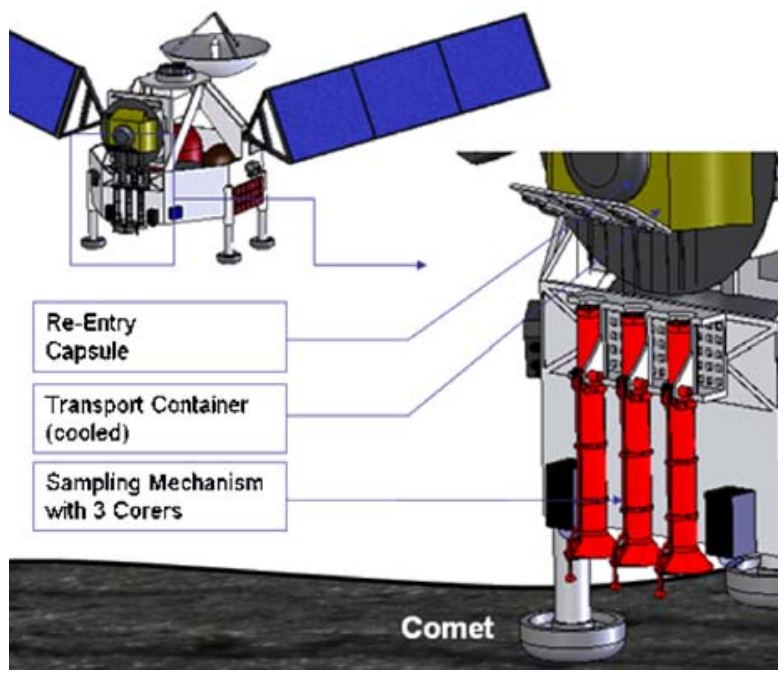


Fig. 6 Penetration depth plotted as a function of time for three different cohesive compression strengths. A friction constant of $0.1 \mathrm{~N} / \mathrm{m}$ is assumed

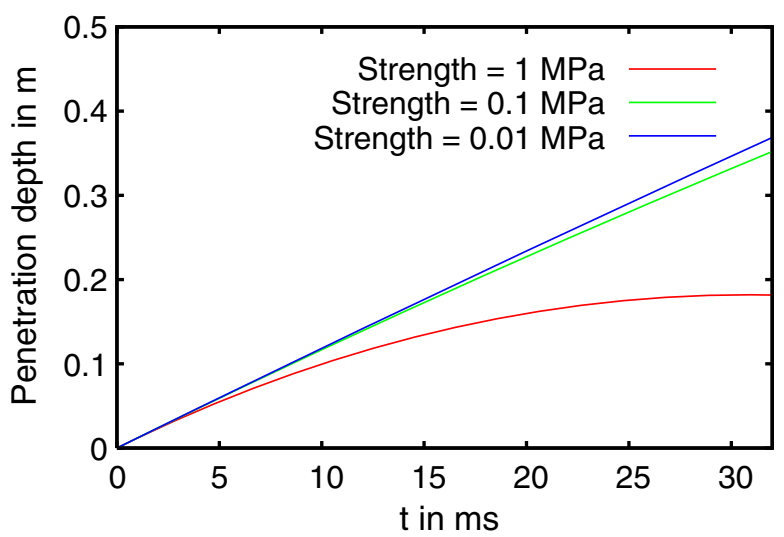

the complete separation of the samples in the cooling compartment. The shutter mechanism is activated by the same cable mechanism that retrieves the coring tube.

The suggested sampling tube will be a Titanium cylinder with a wall thickness of $0.5 \mathrm{~mm}$. Its total weight is about $200 \mathrm{~g}$. Such a cylinder can be accelerated by the springs to a velocity of approximately $12 \mathrm{~m} / \mathrm{s}$ (spring system has flight heritage from the Rosetta lander eject system). Calculations show that the device can penetrate into material of strength of up to approximately $1 \mathrm{MPa}$ (see Fig. 6). The penetration depth is calculated from the linear momentum balance equation taking into account the cohesive force of the material, friction along the surface of the cylinder, gravitational force, and the increase of the moving mass due to shoved material. The calculations represent a lower limit to the actual penetration depth because the cutting force of the sharp edge of the cylinder is not considered. Should the need arise to sample still stronger materials, a projectile gas generator (flight heritage from the Rosetta lander anchor subsystem) could accelerate the same tube to more than $90 \mathrm{~m} / \mathrm{s}$, allowing sampling even of compact water ice.

\subsubsection{Sample analysis strategy}

Handling of samples on Earth, distribution and preservation The future return of extraterrestrial materials by ESA requires a European repository facility. In the present case, it will perform sample separation of gaseous volatiles, ices (including their trapped gases), organics and solid minerals. The gases in the sample containers would be transferred into reservoirs for further analyses. Organics will be present in icy and solid fractions. New techniques are required for separation of these phases under conditions similar to those on the comet. The facility will also coordinate the sample distribution. It will store the major part of the material for posterity, in anticipation of improved future analysis techniques. This facility might be associated with a scientific institution that already has required analytical techniques, experience in micro-sample 
handling including microtome and in situ focused-ion-beam lift-out, as well as the preparation of thin sections. Opening of the containers, sampling and storing of the different phases will be performed in a clean environment, with the sample in vacuum to avoid adsorption of terrestrial atmospheric contaminants.

Examination of the samples with state-of-the-art analytical techniques is necessary to accomplish the major mission goals outlined in Section 2. To maximise scientific output and minimise sample consumption, many techniques will be applied to the same material. Sample requests and allocation (a few percent of the recovered material within the first 5 years after return) should be controlled by a standing committee (similar to NASA's "CAPTEM" committee or its "Meteorite Working Group"). For a fast publication of scientific results, a small fraction $(<1 \%)$ of the returned material will be distributed rapidly to pre-selected research institutions.

Analyses The amount of material returned with Triple F (100 s of grams) will exceed by far that returned with Stardust $(\sim \mathrm{mg})$. Moreover, the sampling technique (Section 3.6.2) will not mix the material intimately with any collector material. These unique mission elements will allow a large number of examinations that were not possible with the Stardust samples.

Initial non-invasive sample inspection could be achieved with x-ray tomography, providing sub-micrometer resolution and full 3D structural information. The measurements will reveal information about porosity, grain size, pore size, stratigraphy and (to some extent) elemental distribution within the sample. Initial mineralogical and petrologic analysis of most of the mineral grains should entail Scanning Electron Microscope (SEM) imaging and Energy Dispersive $\mathrm{X}$-ray (EDX) analyses on all particles, enabling sample requestors to analyse the most suitable samples. Quantitative x-ray spectrometric elemental analyses should be performed on each grain to establish a library of compositions, done by the repository facility for consistency in data. Large particles $(>10 \mu \mathrm{m})$ should be analyzed with synchrotron X-Ray Diffraction (XRD). For smaller particles, microtome slices could be prepared for TEM analysis. Relatively non-destructive spectroscopic analyses including IR, Resonant Raman and UV spectroscopy will follow, prior to further analyses by more or completely destructive techniques, such as, e.g., Secondary Ion Mass Spectroscopy (SIMS) or conventional mass spectrometry.

A major goal of all mineralogical analyses will be the search for high temperature phases and hydrated minerals. The presence of the former would add to the evidence of large scale mixing in the nebula [10,63], whereas the latter would potentially indicate extended warm periods on the comet in the past. Moreover, as yet unknown or fragile minerals might be present that were not preserved in primitive meteorites or Wild 2 particles due to sampling technique or more severe heating on the asteroidal parent bodies.

High-precision mass spectrometric analyses of all fractions of returned cometary material will have highest priority. Deuterium enrichments will unequivocally prove the extraterrestrial origin of the material [44]. H, C, and 
$\mathrm{N}$ isotopic anomalies in the organic fraction will localise interstellar material [11] that has survived the formation of the solar system. The $\mathrm{O}$ isotopic compositions in various extraterrestrial materials exhibit heterogeneities [12], implying spatial or temporal variations during the condensation of the first solar system material. Large samples to be returned with this mission will allow the investigation of refractory elements such as $\mathrm{Mo}, \mathrm{Zr}$ and platinum group elements, which are important to assess heterogeneity and degree of large-scale mixing in the solar system. These analyses require high-precision isotopic measurements, obtainable with MC-ICPMS or TIMS. The samples will also be investigated for stable isotope fractionation effects, e.g. in $\mathrm{Mg}$ and Fe., which will provide information on the conditions during which cometary material formed.

The isotopic compositions of presolar stardust grains indicate that they formed in the dust ejecta of previous star generations [62]. Their abundances in extraterrestrial matter reflect thermal processing in the early solar system and on parent bodies. Thus, the abundance of presolar stardust in returned comet samples, identified by SIMS isotopic mapping, could be a sensitive probe of cometary thermal history. Only a few grains have been identified in comet Wild 2 dust [57], suggesting a lower abundance than in cometary IDPs [48]. However, sampling might have introduced severe bias. The more gentle collection method of this mission will allow presolar grains to be more readily preserved, providing better estimates of the presolar grain abundance in comets. Presolar grains might retain coatings acquired while present in the ISM, but that would not have survived the heating that other extraterrestrial material has experienced.

All cometary matter (ices, organics and minerals) is exposed at various times to irradiation. As the cometary surface will continuously be renewed, the most recent effects of space weathering and the production of cosmogenic nuclides can be examined. Structural changes due to such irradiation, e.g., amorphisation of ices, minerals and carbonaceous matter might be visible. For these studies, noble gases in various grains will be studied, the depth profiles of various elements, as well as the response to irradiation of carbonaceous material with Resonant Raman and IR spectroscopy. The latter techniques will also be used to find evidence for the irradiation of carbonaceous matter in interstellar space prior to its incorporation into the comet. Chemically, isotopically or structurally distinct rims around cometary grains would help to assess the conditions prevailing in the protosolar cloud. The composition of micron-sized grains will be measured, e.g., with the Time of Flight SIMS [58] technique, which provides high sensitivity at a very small scale. This technique also detects organic compounds in situ and provides information about the elemental distributions in mantles around cometary grains.

The volatile (noble gases, $\mathrm{H}, \mathrm{C}, \mathrm{N}, \mathrm{O}, \mathrm{S}$ ) content of cometary ice is controlled by the conditions prevailing during its formation, either in the dense interstellar cloud or in the outer solar nebula [50]. Hence, it is of high priority to determine the relative volatile abundances in the various cometary ice grains. Moreover, the isotopic compositions of noble gases, especially Xe, 
should be determined, both in solid grains and in icy agglomerates, which requires particular, highly sensitive techniques including resonance ionization [26]. Gas abundances of $\mathrm{CH}_{4}, \mathrm{CO}, \mathrm{CO}_{2}, \mathrm{~N}_{2}, \mathrm{NH}_{3}$, etc. are extremely important, e.g., in order to assess the form in which $\mathrm{N}$ has been trapped in the ice, and for comparison with spectroscopic results.

Analyses of organic matter found in ice and associated with mineral grains will provide insights on the early history of our solar system and the extraterrestrial delivery of organic compounds that occurred on early Earth (see Section 2.2). Hence, most important is the search for biologically relevant compounds, such as amino acids or nucleobases, which have been detected in meteorites. In addition, the detection of chiral excesses in these compounds will be essential, as will the comparison with meteoritic organics. Analytical techniques include liquid chromatography with fluorescence detection (HPLC-FD), liquid chromatography-mass spectrometry (LC-MS), gas chromatography-mass spectroscopy (GC-MS) and ionization techniques such as Matrix Assisted Laser Desorption Ionization (MALDI), solid state ${ }^{13} \mathrm{C}$ NMR (Nuclear Magnetic Resonance), XANES (X-ray Absorption Near-Edge Structure) and EPR (Electron Paramagnetic Resonance).

\subsection{Payload}

\section{Overview}

Apart from the sampling device, the mission requires a basic payload to fulfill the following objectives:

1. Characterize the comet, mainly its activity level and topography, with sufficient spatial resolution to identify appropriate sampling sites

2. Mapping of the nucleus to derive important chemical and physical properties that provide the context for the samples

3. Monitor the environment of the spacecraft to avoid hazards, mainly due to cometary dust

An overview of the payload is given in Table 1 . The total mass of that payload is less than $20 \mathrm{~kg}$.

\section{Cameras}

Triple $F$ will carry a complement of four camera systems, designed to address different scientific requirements of the mission. For increased reliability, except for the high resolution camera each of the cameras consists of two identical, cold redundant units, for a total of seven camera heads. The first camera (high resolution imager, HI) provides the global monitoring of the nucleus during comet approach and during phases when the spacecraft is further than several tens of $\mathrm{km}$ from the nucleus. The second camera (the Mapping Imager-MI) will consist of a moderate angular resolution framing imager. This instrument will be responsible for global mapping from distances between 5 and $20 \mathrm{~km}$, corresponding to a resolution between $0.5 \mathrm{~m}$ and $2 \mathrm{~m}$. 
Table 1 Summary of the payload (objective as defined in the text)

\begin{tabular}{llccc}
\hline Instrument & Objective & Mass $(\mathrm{kg})$ & Power $(\mathrm{W})$ & Remarks \\
\hline Cameras & $1,2,3$ & 4.2 & 10 & \\
Near-IR imager or spectrometer & $1,2,3$ & 2.0 & 10 & \\
Thermal IR instrument & 1,2 & 3.5 & 7 & \\
Mass spectrometer & 2 & 4.0 & 10 & Will also examine \\
& & & & small sample \\
Dust in-situ monitoring experiment & 3 & $<5.0$ & 20 & \\
Radio science investigation & 2 & & & \\
Penetrometer & 2 & 0.1 & & On sampling device \\
Permittivity probe & 2 & $<0.3$ & $<1$ & On sampling device \\
Thermal probe & 2 & $<0.1$ & $<0.2$ &
\end{tabular}

The third imager is a miniature wide-angle Fast-Framing Camera (FFC) which will be active during the descent, and ascent phases. This instrument, by acquiring image sequences at a rate in excess of $5 \mathrm{~Hz}$, will document (and possibly guide) the descent of the orbiter with increasing accuracy. In this way the FFC will also provide local context by imaging the neighborhood of the sampling site with a resolution up to about $1 \mathrm{~mm}$. The fourth camera will be a Close-Up Imager (CUI) which will monitor the complete sample acquisition and storage sequence during touch-down. In order to be able to observe the sample in the shadow of the S/C, the CUI will be equipped with a miniature LED illumination device.

The field of view of the HI will be $1.5^{\circ}$, imaged on a 2,048 $\times 2,048$ APS array. The specifications of MI, FFC, and CUI follow those of the cameras in Agnolon [1].

\section{Near-IR imaging spectrometer}

The main scientific objectives of the infrared spectrometer are the following:

- to support the selection of the sampling sites by searching for surface ice and monitoring the gaseous activity on the nucleus and its spatial distribution

- to determine the nature of the solids on the nucleus surface (composition and structure of ices, dust and characterisation of organic compounds)

- to identify the gaseous species in the coma

While it is highly probable that the design of the near-IR instrument will benefit from the technological progress of the future years, we can refer to heritage from instruments already existing or in development.

The proposed instrument could be a simplified version of VIHI, a hyperspectral imager being developed to fly on ESA's BepiColombo. The VIHI channel concept is based on a collecting telescope and a diffraction grating spectrometer ideally joined at the telescope focal plane where the spectrometer entrance slit is located. The image of the slit is dispersed by the diffraction grating onto a two-dimensional detector. A single $256 \times 256$ thinned infrared array detector shall be used which can achieve high quantum efficiency even in the visible domain. The instantaneous acquisition on the two-dimensional 
detector consists of the slit image diffracted by the grating over the selected spectral range $(1-3.5 \mu \mathrm{m})$. The complete image is built in time by subsequent acquisitions (push broom mode). The final result is a three dimensional data set, in which each pixel has a spectrum associated with it. The instantaneous field of view will be $250 \mu \mathrm{rad}$ and the field of view $3.7^{\circ}$. The instrument is housed in a single box that contains the optical system (Telescope plus Spectrometer), the calibration unit, shutter, Focal Plane Assembly (housing the detector and the TE Cooler) and proximity electronics. The operating temperature will be $170 \mathrm{~K}$.

\section{Thermal IR imaging spectrometer/radiometer}

Measuring the thermal emission from the cometary surface would support the selection of the sampling site and provide valuable information about physical and mineralogical characteristics of the target. The temperature of sublimating water ice on the surface is about $200 \mathrm{~K}$ near perihelion. A thermal infrared radiometer (TIR) could be very useful in detecting possible locations of surface ice (and therefore activity) by their low temperature. Furthermore, the TIR spectral range provides a further excellent opportunity to characterize the mineralogical state of the cometary surface.

From the discussion above we propose an integrated imaging TIR spectrometer/radiometer, which works in the 5-40 $\mu \mathrm{m}$ range, be included in the payload of the Triple F mission, an instrument similar to MERTIS on BepiColombo but with a larger $\mathrm{FOV}\left(11.5^{\circ}\right)$ and covering a broader wavelength range for the spectrometer part. Because of the sensitivity limitations of thermal detectors, the spectrometer is designed to provide spectra in 240 spectral channels with good $\mathrm{S} / \mathrm{N}$ mainly for surface temperatures above $300 \mathrm{~K}$ whereas the radiometer is optimized for accurate measurements of the emitted flux in two broadband channels to cover the whole range of relevant temperatures from 100-400 K.

The focal length of the instrument will be around $5 \mathrm{~cm}$. The spatial resolution will be $0.7 \mathrm{mrad}$ for the spectrometer $(7 \mathrm{~m}$ from a distance of $10 \mathrm{~km})$ and $5 \mathrm{mrad}(50 \mathrm{~m}$ from $10 \mathrm{~km})$ for the radiometer. A bolometer and a thermopile will be the detectors for the spectrometer and radiometer, respectively. The total dimension of the instrument will be about $14 \times 16 \times 12 \mathrm{~cm}$.

\section{Mass spectrometer}

It is well known that comets contain a wealth of volatile and super-volatile material including radicals like $\mathrm{CO}, \mathrm{CH}_{2}$, and $\mathrm{CH}_{4}$ (e.g., [3, 24]). The goal of the mission is to bring back original, unprocessed material. However, taking a sample from the comet and bringing it back to the Earth cannot be done without some disturbance to the material, in particular sublimation of volatiles. Therefore, it is mandatory to have a mass spectrometer on board, which can analyze the most volatile or reactive compounds in situ in order to get the starting condition of the sample. The measurement would be twofold: the mass spectrometer would analyze the natural outgassing of the comet in the approach and prelanding phase. When acquiring the main sample a second much smaller sample will be acquired and placed in front of the mass 
spectrometer. The mass spectrometer can then analyze the composition of this second sample during the journey back to the Earth over a long time (until the sample has fully evaporated). This will require two separate inlets to the ion source which can easily be achieved because the direction of the neutral gas flow is not critical. The mass spectrometer will not have the same resolution and sensitivity as laboratory mass spectrometers, but it will allow us to assess the initial condition of the sample and to deduce the chemical alterations the material has undergone from the comet to the laboratories on Earth.

We foresee using a time of flight mass spectrometer with an electron bombardment ion source. Such an instrument can easily reach a mass resolution $\mathrm{m} / \mathrm{dm}$ of 1,500 , high sensitivity and mass ranges up to $>300 \mathrm{amu}$. The design could be based on the heritage of Rosetta-ROSINA/RTOF [4].

However, RTOF is too heavy to be included in the present mission. This can easily be solved by omitting the requirement that the mass spectrometer has to be built with an ultra-high vacuum enclosure and be launched sealed under vacuum as was done for ROSINA/RTOF. This requirement was necessary in the case of the Rosetta mission to study the comet far away from the Sun when cometary activity is minimal. RTOF has two independent channels (ion sources and detectors) that can be reduced to one for the present mission, thus saving not only detector and ion source but also electronics. Therefore, it would be possible, with current technology, to build a reflectron-time of flight instrument with a mass budget of $4 \mathrm{~kg}$. The dimensions of the instrument will be about $20 \times 20 \times 50 \mathrm{~cm}$.

\section{Dust in-situ monitoring experiment}

The S/C will have to operate deep inside the comet coma, both during the monitoring phase and during the descent for sampling. Therefore, it is important to characterize the dust distribution in the coma in order to control and guarantee safety for mission operation and $\mathrm{S} / \mathrm{C}$ health. Therefore, an in situ dust instrument is essential as a "security device" to measure the dust flux on the $\mathrm{S} / \mathrm{C}$.

The GIADA experiment on board Rosetta will study the cometary dust environment of 67P and will accomplish unprecedented in situ primary scientific measurements [13]. A similar experiment on board the Triple $F$ mission will fulfill the aforementioned security requirements and will in addition provide scientific data as it shall provide real time data on dust flux of "direct" and "reflected" grains, dust velocity distribution, dust evolution in the coma, dust changes vs. nucleus evolution and emission anisotropy, determination of dust-to-gas ratio, and identification of non homogeneous dust emission features from the surface (active areas, jets).

The instrument is designed to measure momentum, scalar velocity and mass of single grains. From the detection of particles vs. time information about dust abundance and spatial distribution vs. physical and dynamic properties is derived. Its two stages of detection form a cascade: an Optical Detection 
System (ODS) and an Impact Sensor (IS). When a grain crosses the ODS, it is optically (scattered/reflected signal) detected, and its physical (shape and size, mainly) and chemical (through the optical constants) properties are measured. For each grain impacting onto the IS, the momentum of the incident grain is measured. With this system it is possible to measure abundance and dynamic properties of grains present in the comet coma. The ODS gives a first estimate of the speed of each crossing grain, and the speed of the grain is measured again, but with more accuracy, from the time-of-flight between ODS and IS. In this way for each detected grain, speed, time-of-flight, momentum and, therefore, mass are measured. The field of view of the ODS + IS system is about $40^{\circ}$. In addition, a network of microbalances (MBSs), with field of view of about $40^{\circ}$ each, point in different directions to monitor flux of submicron and micron particles from $10^{-10}$ to $10^{-4} \mathrm{~g}$. The instrument is equipped with controlling electronics to drive the sensors and to transmit telecommunication to and from the spacecraft. The size of the instrument is about $23 \times 25 \times 30 \mathrm{~cm}$.

\section{Radio science investigation}

The Radio Science Experiment RSE will use the radio signals transmitted from the onboard radio subsystem at the carrier frequencies X-band and Ka-band in the two-way radio mode (X-band uplink). The goal is to derive perturbing forces acting on the spacecraft by measuring the Doppler shift of the carrier signals caused by additional changes in relative velocity. Perturbing forces of interest may be:

- the gravity acceleration of the cometary nucleus which would reveal mass, bulk density (together with a volume estimate from the camera)

- the outgassing from the cometary nucleus which would reveal the combined gas and dust production rate (although the major contribution will come from the gas).

The measurements require a highly stable two-way link where the stability of the X-band uplink is derived from the ground station's hydrogen maser. The spacecraft receives the X-band uplink and transponds it back to Earth phasecoherently at two simultaneous downlink frequencies at X-band and Ka-band. Although the Ka-band downlink seems to be much noisier than the X-band downlink, it is possible to correct for the plasma noise in order to achieve a clear and four times stronger Doppler signal at Ka-band than at X-band. This allows us the detection of very small perturbations in the $<10 \mu \mathrm{m} / \mathrm{s}$ range. $\mathrm{X} / \mathrm{X}$ and $\mathrm{X} / \mathrm{Ka}$ transponders are available on the market and have been flown on ESA missions (SMART-1).

These measurements require that the spacecraft performs attitude changes using the reaction wheels only during the gravity observations. Any attitude changes by thruster firing would destroy the observations. 
The mass determination shall be an iterative process starting during the approach phase. Each mass determination at a certain error level will allow manoeuvring the spacecraft closer to the nucleus.

\section{Comet Penetrometry Experiment (CPEx)}

Penetrometry is the use of a penetrating probe to measure mechanical properties of the target material. A comet nucleus sample return mission offers a rare opportunity to make contact with undisturbed cometary subsurface material. Compared with previous missions, Triple $F$ will provide measurements at multiple locations on the target comet. Penetrometry at the sampling locations will be useful for two main reasons:

Firstly, it can support sample collection by means of dynamics measurements performed during sampling. Questions that may be addressed include: Where did the material actually come from? What was its undisturbed volume? What is the strength and texture of the undisturbed material? To what extent was it disturbed, crushed and mixed during sampling? Secondly, penetrometry can generate unique ground-truth science. The penetration resistance of cometary material is sensitive to its origin and modification. Furthermore, measurements with depth can detect layering, e.g. the presence of low-cohesion material overlying a sintered layer at the water ice sublimation front. European heritage in this technique is strong, including sensors flown on Huygens and Philae.

Although the primary requirement for sampling limits the possibilities for dedicated geotechnical measurements, a great deal may still be learned from a set of simple measurements. The solution we propose for Triple $F$ is to incorporate small sensors to monitor dynamics during touchdown and sampling. Assuming a short-duration (few s) touchdown and a coring tube driven quickly (10 m/s initial speed) into the ground, we propose the following:

- Displacement sensor to monitor the relative position of the sampling tube and spacecraft. This could be implemented by means of an optical barcode technique. Stripes affixed to the core tube would be interrogated by a small light source and photodiode. Mass: $\sim 5 \mathrm{~g}$. Data: 1 bit at $50 \mathrm{kHz}$ during sampling, i.e. $50 \mathrm{kbit} / \mathrm{s}$. Heritage: $\mathrm{HP}^{3}$ DACTIL tether length sensor.

- Microphone-acoustic vibrations in the sampling mechanism should be sensitive to the texture of the sampled material and its variation with depth. A small piezoelectric or strain gauge sensor mounted on the sampling system at a location affording good acoustic coupling could fulfill this role. Mass: $\sim 2$ g. Data: 12 bits at $50 \mathrm{kHz}$ during sampling, i.e. $600 \mathrm{kbit} / \mathrm{s}$. Heritage: COTS aerospace components.

- If possible, a single-axis accelerometer mounted near the upper end of the coring tube. This would measure the deceleration, and thus force, encountered by the sampling tube. Mass $\sim 2 \mathrm{~g}$. Data: 12 bits at $50 \mathrm{kHz}$ during sampling, i.e. $600 \mathrm{kbit} / \mathrm{s}$. Heritage: COTS aerospace components, Philae MUPUS ANC-M, Huygens SSP ACC-I, Huygens HASI ACC, HP 3 DACTIL. 
- Harness and electronics: $100 \mathrm{~g}$ for the displacement, microphone and accelerometer sensors.

Permittivity probe

A permittivity probe measuring the electric properties of the sample between $0.1 \mathrm{~Hz}$ and $10 \mathrm{kHz}$ is well suited to monitor changes in internal structure of the sample which are associated with sublimation processes of $\mathrm{H}_{2} \mathrm{O}$. The electrical permittivity of ice is unique amongst the rock forming minerals in the low frequency range, as the dielectric constant is of the order of 100 under static conditions, thus, more than an order of magnitude higher than for rocky materials, and decreases with increasing frequency to a value of 3.1 for infinite frequency.

The permittivity probe used to monitor the sample closely follows the design of a mutual impedance probe. Ringsector-like electrodes printed onto a Kapton foil are attached at different depths to the inner wall of the sample container. A current generator is connected to two selectable transmitter electrodes, and the generated voltage is sensed as a function of frequency by a number of receiver electrodes. By using different transmitter/receiver geometries across the sample an electrical "image" of the sample can be created. The total mass of the permittivity probe including electronics and harness is estimated to be less than $300 \mathrm{~g}$ and the average power consumption during operation is less than $1 \mathrm{~W}$.

\section{Thermal probe on sampling device}

The knowledge of the original cometary temperature profile at the time of sampling measured as a function of depth would be of considerable value for the interpretation of the sample measurements. Because the titanium sample container has a several orders of magnitude higher thermal conductivity than the cometary soil, contact measurements cannot easily be used for that purpose. A possible solution is, therefore, based on the use of radiometric sensors (e.g. thermopiles as will be used for measuring the surface temperature at the Rosetta PHILAE landing site of comet 67P/Churyumov-Gerasimenko, [56]) which are mounted in different depths at the inner wall of the sample container. The short time constant of these sensors of about 200 ms makes the measurement of the temperature profile during the first seconds after insertion of the sampling device possible. This is before the temperature of the low conductivity cometary material can effectively adjust to the wall temperature. In addition, continuing the measurements after sampling would allow inferring thermal properties of the fresh sample. Furthermore, in case that a significant amount of heat is released inside the sample in the process of crystallization of originally amorphous ice, monitoring of the sample temperature as a function of depth could prove the existence of this process. Assuming 25\% amorphous ice in the lower parts of the sample, the crystallization heat would increase the temperature by approximately $20 \mathrm{~K}$, which is easily detectable by the sensors.

For the reasons described above the implementation of several (around 8 -16) thermopile sensors into the sampling device could provide valuable 
information about the initial temperature profile of the sample. The resources needed by this instrumentation are minor, basically an electronics comprising of multiplexer, amplifier and ADC (assuming that another DPU controls the data acquisition) with a mass of about $100 \mathrm{~g}$ (including harness) and a power consumption of less than $200 \mathrm{~mW}$.

\section{Summary}

A Comet nucleus sample return mission is a unique means to provide valuable information about

- The formation of the solar system from the protosolar nebula

- The role comets played for the development of the hydrosphere and life on Earth

- The way cometary activity works

Recent results from the Deep Impact mission show that the cometary surface is of low strength and that cold, relatively unprocessed material can be found at shallow depths of a few tens of cms. Therefore, the return of a cooled sample from an active Jupiter-family comet is possible as a mediumsized mission with a total duration of approximately 10 years.

The Triple $F$ mission has not been chosen for the first cycle of Cosmic Vision. Nevertheless, we believe that it is a compelling mission concept that should be considered for future space exploration programs.

Open Access This article is distributed under the terms of the Creative Commons Attribution Noncommercial License which permits any noncommercial use, distribution, and reproduction in any medium, provided the original author(s) and source are credited.

\section{References}

1. Agnolon, D.: ESA Technology Reference study report SCI-PA/2007/004/DA (2007)

2. A'Hearn, M.F., et al.: Science 310, 258 (2005)

3. Altwegg, K., Balsiger, H., Geiss, J.: Astron. Astrophys. 290, 318 (1994)

4. Balsiger, H., et al.: Space Sci. Rev. 128, 745 (2007)

5. Bar-Nun, A., Laufer, D.: Icarus 161, 157 (2003)

6. Belton, M.J.S., et al.: Icarus 187, 332 (2007)

7. Bizzarro, M., et al.: Science 316, 1178 (2007)

8. Blum, J., et al.: Astrophys. J. 652, 1768 (2006)

9. Brown, M.E., et al.: Nature 446, 294 (2007)

10. Brownlee, D.E., et al.: Science 314, 1711 (2006)

11. Busemann, H., et al.: Science 312, 727 (2006)

12. Clayton, D.: In: Davies, A.M. (ed.) Treatise on Geochemistry-Meteorites, comets, and planets, vol. 1, pp. 269. Elsevier (2003)

13. Colangeli, L., et al.: Space Sci. Rev. 128, 803 (2007)

14. Davidsson, B.J.R., Gutiérrez, P.J., Rickman, H.: Icarus 187, 306 (2007)

15. Delsemme, A.H.: American Scientist. 89, 432 (2001)

16. Drake, M.J.: Meteorit. Planet. Sci. 40, 519 (2005)

17. Duncan, M., Levison, H., Dones, L.: In: Comets, II, Festou, M.C., Keller, H.U., Weaver, H.A. (eds.) The Univ. of Arizona Press (Tucson), pp. 193 (2004) 
18. Ehrenfreund, P., et al.: Rep. Prog. Phys. 65, 1427 (2002)

19. Farinella, P., Davis, D.R.: Science 273, 5277 (1996)

20. Feaga, L.M., et al.: Icarus 190, 345 (2007)

21. Fray, N., et al.: Icarus 184, 239 (2006)

22. Fujiware, A., Mukai, T., Kawaguchi, J., Uesugi, K.T.: Adv. Space Res. 25, 231 (2000)

23. Gardinier, A., et al.: Earth Planet Sci. Lett. 184, 9 (2000)

24. Geiss, J., et al.: Space Sci. Rev. 90, 253 (1999)

25. Gerakines, P.A., Moore, M.H., Hudson, R.: Icarus 170, 202 (2004)

26. Gilmour, J.D., et al.: Rev. Sc. Instrum. 65, 617 (1994)

27. Glassmeier, K.-H., et al.: Space Sci. Rev. 128, 1 (2007)

28. Goesmann, F., et al.: Space Sci. Rev. 128, 257 (2007)

29. Groussin, O., et al.: Icarus 187, 16 (2007)

30. Grün, E., et al.: J. Geophys. Res. 98, 15091 (1993)

31. Holsapple, K.A., Housen, K.R.: Icarus 187, 345 (2007)

32. Keller, H.U., et al.: Nature 321, 320 (1986)

33. Keller, H.U., et al.: Astron. Astrophys. 187, 807 (1987)

34. Keller, H.U., Britt, D., Buratti, B.J., Thomas, N.: In: Comets II, Festou, M.C., Keller, H.U., Weaver, H.A. (eds.) The Univ. of Arizona Press (Tucson), pp. 211 (2004)

35. Kossacki, K., Szutowicz, S.: Icarus 1995, 705 (2008)

36. Kührt, E.: Earth Moon, Planets 90, 61 (2002)

37. Kührt, E., Keller, H.U.: Icarus 109, 121 (1994)

38. Laufer, D., Pat-El, I., Bar-Nun, A.: Icarus 178, 248 (2005)

39. Lowry, S.C., et al.: Astron. Astrophys. 349, 649 (1999)

40. Marcano, V., Benitez, P., Palacios-Prü, E.: Planet. Space Sci. 51, 159 (2003)

41. Marty, B., et al.: Science 319, 75 (2008)

42. McKeegan, K.D., et al.: Science 314, 1724 (2006)

43. Mennella, V., et al.: Astrophys. J. 507, L177 (1998)

44. Messenger, S.: Nature 404, 968 (2000)

45. Morbidelli, A., et al.: Meteorit. Planet Sci. 35, 1309 (2000)

46. Nelson, R.M., Rayman, M.D., Weaver, H.A.: Icarus 167, 1 (2004)

47. Ng, T.C., et al.: Lunar Planetary Sci. Conf. 34, abstract \#1002 (2003)

48. Nguyen, A.N., Busemann, H., Nittler, L.R.: Lunar Planetary Sci. Conf. 38, abstract \#2332 (2007)

49. Owen, T.C., Bar-Nun, A.: Orig. Life Evol. Biosph. 31, 435 (2001)

50. Owen, T.C.: Space Sci. Rev. (2008, in press). doi:10.1007/s11214-008-9306-7

51. Pepin, R.O.: Earth Planet. Sci. Lett. 252, 1 (2006)

52. Porcelli, D., Woolum, D., Cassen, P.: Earth Planet. Sci. Lett. 193, 237 (2001)

53. Reinhard, R., Battrick, B. (eds.): Space Missions to Halley's Comet, ESA-SP 1066 (1986)

54. Sandford, S.A., et al.: Science 314, 1720 (2006)

55. Soderblom, L.A., et al.: Science 296, 1087 (2002)

56. Spohn, T., et al.: Space Sci. Rev. 128, 339 (2007)

57. Stadermann, F.J., Floss, C.: Lunar Planet. Sc. Conf., abstract \#1889 (2008)

58. Stephan, T.: Planet. Space Sci. 49, 859 (2001)

59. Sunshine, J.M., et al.: Science 311, 1453 (2006)

60. Sunshine, J.M., et al.: Icarus 190, 284 (2007)

61. Thomas, P.C., et al.: Icarus 187, 4 (2007)

62. Zinner, E.: In: Davies, A.M. (ed.) Treatise on Geochemistry-Meteorites, comets, and planets, vol. 1, p. 17. Elsevier (2003)

63. Zolensky, M.E., et al.: Science 314, 1735 (2006) 